\title{
In Vitro Assessment of the Drug-Drug Interaction Potential of Verinurad and Its Metabolites as Substrates and Inhibitors of Metabolizing Enzymes and Drug Transporters ${ }^{\Phi}$
}

\author{
V. Sashi Gopaul, Anna Vildhede, Tommy B. Andersson, Fredrik Erlandsson, Caroline A. Lee, ${ }^{1}$ \\ Susanne Johansson, ${ }^{*}$ and Constanze Hilgendorf* \\ Early Research and Development Cardiovascular Renal and Metabolism, AstraZeneca Biopharmaceuticals R\&D Gothenburg, \\ Mölndal, Sweden (V.S.G, A.V., T.B.A, C.H.); CVRM Late Clinical, AstraZeneca Biopharmaceuticals R\&D Gothenburg, Mölndal, \\ Sweden (F.E.); Ardea Biosciences, San Diego, CA, USA (C.A.L.); Clinical Pharmacology \& Safety Sciences \& AstraZeneca \\ Biopharmaceuticals R\&D Gothenburg, Mölndal, Sweden (S.J.)
}

Received Feb 03, 2021; accepted May 24, 2021

\begin{abstract}
Verinurad is a selective uric acid transporter 1 (URAT1) inhibitor in development for the treatment of chronic kidney disease and heart failure. In humans, two major acyl glucuronide metabolites have been identified: direct glucuronide $\mathrm{M} 1$ and $\mathrm{N}$-oxide glucuronide M8. Using in vitro systems recommended by regulatory agencies, we evaluated the interactions of verinurad, M1, and $M 8$ with major drug-metabolizing enzymes and transporters and the potential for clinically relevant drug-drug interactions (DDIs). The $\mathrm{IC}_{50}$ for inhibition of CYP2C8, CYP2C9, and CYP3A4/5 for verinurad was $\geq 14.5 \mu \mathrm{M}$, and maximum free plasma concentration $\left(\mathrm{I}_{\mathrm{u}, \mathrm{max}}\right) / \mathrm{IC}_{50}$ was $<0.02$ at the anticipated therapeutic $\mathrm{C}_{\max }$ and therefore not considered a DDI risk. Verinurad was not an inducer of CYP1A2, CYP2B6, or CYP3A4/5. Verinurad was identified as a substrate of the hepatic uptake transporter organic anion-transporting polypeptide (OATP) 1B3. Since verinurad hepatic uptake involved both active and passive transport, there is a low risk of clinically relevant DDIs with OATP, and further study is warranted. Verinurad was a substrate of the efflux transporters P-glycoprotein $(P-g p)$ and breast cancer resistance protein (BCRP), and renal transporter
\end{abstract}

organic anion transporter 1 (OAT1), although it is not considered a DDI risk in vivo because of dose-proportional pharmacokinetics (P-gp and BCRP) and limited renal excretion of verinurad (OAT1). M1 and M8 were substrates of multidrug resistance-associated protein (MRP) 2 and MRP4 and inhibitors of MRP2. Apart from verinurad being a substrate of OATP1B3 in vitro, the potential for clinically relevant DDls involving verinurad and its metabolites as victims or perpetrators of metabolizing enzymes or drug transporters is considered low.

\section{SIGNIFICANCE STATEMENT}

Drug transporters and metabolizing enzymes have an important role in the absorption and disposition of a drug and its metabolites. Using in vitro systems recommended by regulatory agencies, we determined that, apart from verinurad being a substrate of organic anion-transporting polypeptide 1B3, the potential for clinically relevant drug-drug interactions involving verinurad and its metabolites M1 and M8 as victims or perpetrators of metabolizing enzymes or drug transporters is considered low.
This work and development of this manuscript was supported by AstraZeneca.

V. Sashi Gopaul, Anna Vildhede, Fredrik Erlandsson, Susanne Johansson, and Constanze Hilgendorf are employees of AstraZeneca. Caroline A. Lee is a former employee of Ardea Biosciences, a subsidiary of AstraZeneca. Tommy B. Andersson is a former employee of AstraZeneca.

${ }^{1}$ Current affiliation: Arena Pharmaceuticals, Inc., San Diego, CA, USA

S.J. and C.H. contributed equally to this work.

https://dx.doi.org/10.1124/jpet.121.000549.

[S This article has supplemental material available at jpet.aspetjournals. org.

\section{Introduction}

Verinurad is a novel, selective inhibitor of URAT1 that was initially studied for the treatment of gout (Shiramoto et al., 2018; Fitz-Patrick et al., 2019; Terkeltaub et al., 2019). Verinurad is currently in development for chronic kidney disease (CKD) and heart failure with preserved ejection fraction (HFpEF), in which high levels of uric acid are observed and may constitute either a contributing cause or risk factor for

ABBREVIATIONS: AGP, $\alpha$-1-acid glycoprotein; ATP, adenosine triphosphate; AUC, area under the curve; BCRP, breast cancer resistance protein; BSEP, bile salt export pump; $\mathrm{CHO}$, Chinese hamster ovary; $\mathrm{CKD}$, chronic kidney disease; $\mathrm{C}_{\max }$, maximum observed plasma concentration; $\mathrm{C}_{\mathrm{u} \text {,max }}$, maximum free plasma concentration; DDI, drug-drug interaction; EMA, European Medicines Agency; ER, efflux ratio; FDA, Food and Drug Administration; HLM, human liver microsome; HSA, human serum albumin; I $I_{\max }$, total maximum plasma inhibitor concentration; $\mathrm{I}_{\mathrm{u}, \mathrm{max}}$, maximum free plasma concentration; JPMDA, Japanese Pharmaceuticals and Medical Devices Agency; $\quad \mathrm{KCl}$, potassium chloride; LC-MS/MS, liquid chromatography with tandem mass spectrometry; MATE, multidrug and toxin extrusion; MDCK, Madin-Darby Canine Kidney; MOPS, 4-morpholinepropanesulfonic acid; MRP, multidrug resistance-associated protein; NADPH, nicotinamide adenine dinucleotide phosphate; NMR, nuclear magnetic resonance; OAT, organic anion transporter; OATP, organic anion-transporting polypeptide; OCT, organic cation transporter; CYP, cytochrome P450; $\mathrm{P}_{a p p}$, apparent permeability; P-gp, P-glycoprotein; PPB, plasma protein binding; Sf9, Spodoptera frugiperda; TDI, time-dependent inhibition; UGT, glucuronosyl transferase; URAT1, uric acid transporter 1. 
the diseases (Ramirez-Sandoval and Madero, 2018; Sato et al., 2019; Carnicelli et al., 2020a,b). Multiple clinical trials have studied the pharmacokinetics, pharmacodynamics, safety, and tolerability of verinurad (Shen et al., 2017; Hall et al., 2018; Lee et al., 2018). Based on its potent inhibition of URAT1 (25 nM) (Tan et al., 2017), pharmacokinetic properties in humans (Shen et al., 2017), as well as high permeability and solubility, the expected therapeutic dose of verinurad is low $(<30 \mathrm{mg} /$ day). Verinurad is currently being evaluated in two separate phase 2 studies in patients with CKD (NCT03990363) or heart failure (NCT04327024) at doses up to $24 \mathrm{mg}$ once daily.

Verinurad shows dose-proportional exposure (maximum observed plasma concentration $\left[\mathrm{C}_{\max }\right]$ and area under the curve [AUC]) up to and including $40 \mathrm{mg}$ following a single dose and up to and including $15 \mathrm{mg}$ following multiple oncedaily doses (Shen et al., 2017; Hall et al., 2018). Furthermore, there is minimal accumulation of verinurad (approximately 1.2-fold for $\mathrm{C}_{\max }$ and 1.3-fold for AUC) observed after oncedaily dosing (Hall et al., 2018). Verinurad is primarily metabolized by multiple glucuronosyl transferases (UGT1A1, 1A3, 1A8, 2B4, 2B7, and 2B17) and cytochrome P450 CYP enzymes CYP3A4 and CYP2C8 (Lee et al., 2018). The acyl glucuronide metabolites M1 (direct glucuronidation) and M8 (glucuronidation of $N$-oxide) (Fig. 1) are rapidly formed after the absorption of verinurad and circulate in the plasma at concentrations equimolar to verinurad but lack efficacy toward inhibition of URAT1 (Lee et al., 2018). M1 is formed by direct glucuronidation of verinurad via several UGTs. The formation of the $N$ oxide metabolite of verinurad (M4) is mediated by CYP3A4 in
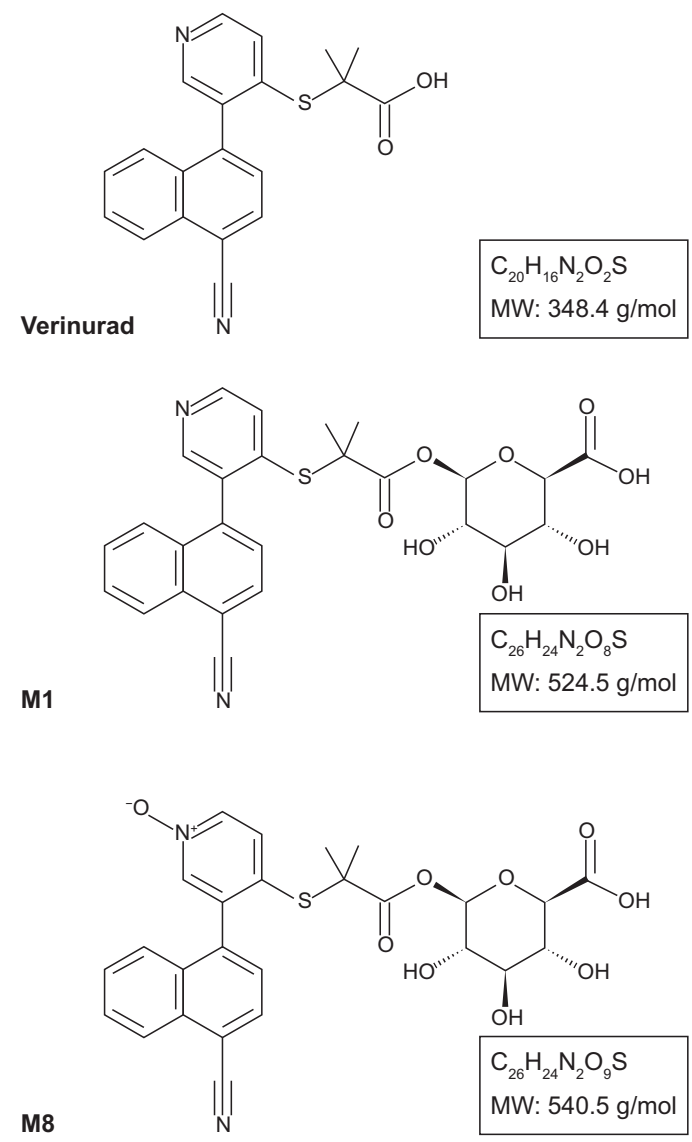

Fig. 1. Structures of verinurad and metabolites M1 and M8. MW, molecular weight. vitro, which is further metabolized by UGTs to form M8 (Lee et al., 2018). Additionally, M8 can be formed from M1 via CYP2C8. Therefore, as reported previously (Lee et al., 2018), the potential for comedications to alter the exposure of verinurad as a substrate of UGT and CYP enzymes is low due to the involvement of multiple parallel and sequential metabolic pathways. While verinurad is excreted via the feces, metabolites M1 and M8 are excreted renally.

Based on observations of enterohepatic recirculation, it is hypothesized that the disposition of verinurad and its metabolites involves active hepatic uptake and renal secretion (Lee et al., 2018). Given the importance of transporters in the absorption and disposition of a drug and its metabolites, this can contribute to clinically relevant drug-drug interactions (DDIs) (Varma et al., 2015; Zhang et al., 2018). In particular, the involvement of multiple mechanisms in the disposition of drugs may lead to mechanistically complex DDIs (Varma et al., 2015). For this reason, the evaluation of pharmacokinetic DDIs of new molecular entities requires multiple methodologies that combine in vitro, in silico, and clinical studies early in drug development and following the most recent guidelines issued by regulatory agencies (European Medicines Agency, 2013; Japanese Pharmaceuticals and Medical Devices Agency, 2018; Food and Drug Administration, 2020).

By virtue of its mechanism of action of URAT1 inhibition, verinurad shows a pharmacodynamic-related DDI when coadministered with allopurinol. The active metabolite of allopurinol, oxypurinol, is a substrate for URAT1 and oxypurinol reabsorption, and thereby exposure decreases when given with verinurad (Kankam et al., 2018).

Here, we adopt a comprehensive strategy using a range of in vitro systems to determine the in vitro interactions of verinurad and its major metabolites M1 and M8 as victims or perpetrators of drug transporters and metabolizing enzymes (CYPs and UGTs). The in vitro studies reported here were conducted with appropriate reference inhibitors and positive controls in line with the acceptance criteria for assay performance according to recently published guidance (European Medicines Agency, 2013; Japanese Pharmaceuticals and Medical Devices Agency, 2018; Food and Drug Administration, 2020). The clinical relevance of the in vitro findings is further contextualized within the framework of basic static equations to evaluate the potential for DDIs, following guidance provided by regulatory agencies to identify potential clinical DDIs and avoid adverse clinical consequences (European Medicines Agency, 2013; Japanese Pharmaceuticals and Medical Devices Agency, 2018; Food and Drug Administration, 2020).

\section{Materials and Methods}

Materials. Unlabeled verinurad (2-(3-(4-cyanonaphthalen-1-yl)pyridin-4-ylthio)-2-methylpropanoic acid) was synthesized at Piramal Pharma Solutions (Torcan, Canada) following the synthetic route described previously (Ouk, 2013; Lee et al., 2018). ${ }^{14} \mathrm{C}$-verinurad ethanol solution $(0.5 \mathrm{mCi} / \mathrm{ml})$ with a specific activity of $55.2 \mathrm{mCi} / \mathrm{mol}$ and radiochemical purity of $98.8 \%(3.2 \mathrm{mg} / \mathrm{ml})$ was obtained from Moravek Biochemicals, Inc. (Brea, CA).

Verinurad acyl glucuronide (M1), verinurad $N$-oxide acyl glucuronide (M8), and the $\mathrm{D}_{6}$-stable isotope-labeled verinurad were synthesized by Ardea Biosciences, Inc. (part of AstraZeneca, San Diego, CA), and the $\mathrm{D}_{6}$-stable isotope-labeled metabolites for M1 and M8 were synthesized by Syncom BV (Groningen, The Netherlands), as described previously (Lee et al., 2018). The identity of the materials 
was confirmed by liquid chromatography-mass spectrometry and nuclear magnetic resonance (NMR), and the purity was determined by quantitative NMR.

All other materials, such as solvents, buffer salts, or reference compounds, were of high-performance liquid chromatography or analytical grade.

Plasma Protein Binding. In vitro plasma protein binding (PPB) of verinurad was evaluated in pooled $(n>3)$ human plasma (BioIVT, Westbury, NY), human serum albumin (HSA; Sigma-Aldrich, St Louis, MO), and $\alpha$-1-acid glycoprotein (AGP; Sigma-Aldrich) in triplicate. Plasma and protein solutions were spiked with ${ }^{14} \mathrm{C}$-verinurad to achieve concentrations of 1,10 , and $50 \mu \mathrm{M}$. Incubations were conducted at $37^{\circ} \mathrm{C}$ for 18 hours to allow for equilibrium of free drug to be reached between the plasma and buffer chambers using the rapid equilibrium dialysis device (Thermo Fisher Scientific, Waltham, MA). Sample aliquots from each chamber were analyzed by liquid scintillation counting. Radioactivity concentrations in aliquots from both sample and buffer chamber were determined directly in $6 \mathrm{ml}$ Ultima Goldcocktail (Perkin-Elmer, Waltham, MA) using a Packard Tri-Carb 3100TR liquid scintillation counter (Perkin-Elmer).

Due to the difference in stability of the acyl glucuronide metabolites compared with the parent drug, the PPB of metabolites M1 and M8 was determined in pooled ( $n>3)$ human mixed-sex plasma (BioIVT) by ultrafiltration using the Ultrafiltration Device with Ultracel YM-T membrane (Merck-Millipore, 4104). The stability of glucuronides M1 and M8 under assay conditions was verified prior to the PPB assay. M1 or M8 were added to $500 \mu \mathrm{L}$ human plasma at a final concentration of 1 and $50 \mu \mathrm{M}$, with the final volume containing $1 \%$ of organic solvent. An aliquot of $50 \mu \mathrm{l}$ taken immediately served as the $\mathrm{T}=0$ minute sample. The remaining solution was incubated at $37^{\circ} \mathrm{C}$ for 30 minutes. A total of $300 \mu \mathrm{L}$ plasma sample was loaded into the Ultrafiltration Device and centrifuged at $37^{\circ} \mathrm{C}$ at $2000 \mathrm{~g}$ for 10 minutes. At the end of centrifugation, $50 \mu \mathrm{L}$ filtrate was analyzed, and the remaining volume of filtrate in each collection tube was measured to determine the total volume of filtrate for recovery calculation. M1 and M8 concentrations in filtrate and stability samples were analyzed by liquid chromatography with tandem mass spectrometry (LC-MS/MS) (Supplemental Table 1).

Enzyme Systems. Pooled human liver microsomes (HLMs; 150 donors, mixed sex) for in vitro enzyme inhibition studies were purchased from BD Biosciences (San Jose, CA, USA) and Corning Corporation (Corning, NY, USA). Fresh primary human hepatocytes $(n=3$ different female donors) for in vitro induction studies were obtained from XenoTech LLC (Kansas City, KS).

In Vitro Evaluation of Verinurad, M1, and M8 as Inhibitors of Human Metabolizing Enzymes. An evaluation of verinurad and metabolites M1 and M8 as reversible and time-dependent inhibitors of human CYP enzymes, as well as verinurad as a reversible inhibitor of human UGT1A1 and UGT2B7, was performed in pooled HLMs. Details of the incubation are provided in Supplemental Table 2. All reactions were performed in triplicate. The final dimethyl sulfoxide (DMSO) concentration in the incubations was $\leq 0.3 \%$.

Reversible enzyme inhibition was investigated by coincubation of enzyme marker substrates (Supplemental Table 2) with HLMs in the presence of different concentrations of test compound (verinurad, M1, or M8) and either $1 \mathrm{mM}$ nicotinamide adenine dinucleotide phosphate (NADPH) for CYP enzymes or $5 \mathrm{mM}$ uridine diphosphate glucuronic acid for UGT enzymes. Incubations with a known inhibitor for each enzyme served as a positive control. Reactions were terminated by the addition of ice-cold acetonitrile containing internal standard, and proteins were precipitated by centrifugation. The resulting supernatant was analyzed by LC-MS/MS to monitor the rate of formation of CYP isoform-specific metabolites (Supplemental Table 1).

Assessment of time-dependent inhibition (TDI) of CYP enzymes by verinurad was performed using a 30-minute preincubation of test compound at two different concentrations with HLMs and cofactors and then following the protocol for the reversible inhibition assay as described above. Assessment of the potential for TDI of CYP enzymes by M1 and M8 included a 30-minute preincubation with HLMs at two different concentrations $(10$ and $50 \mu \mathrm{M})$ in the presence and absence of $1 \mathrm{mM}$ NADPH followed by a 10-fold dilution and incubation in the presence of CYP enzyme marker substrates and $1 \mathrm{mM}$ NADPH.

In Vitro Evaluation of Verinurad as a CYP Inducer in Primary Human Hepatocytes. The evaluation of verinurad as an inducer of CYP1A2, CYP2B6, and CYP3A4/5 was performed in sandwich-cultured human hepatocytes from three individual donors. Details of the incubation are provided in Supplemental Table 3. Briefly, hepatocytes were treated once daily for three consecutive days with either vehicle control (0.1\% DMSO), verinurad (1, 10, or $100 \mu \mathrm{M})$, or one of three prototypical CYP inducers. Approximately 24 hours after the last treatment, the cells were incubated with enzyme marker substrates for 30 minutes at $37^{\circ} \mathrm{C}$ to monitor the rate of formation of CYP isoform-specific metabolites (LC-MS/MS; Supplemental Table 1). Additional hepatocytes from the same treatment groups were harvested with TRIzol to isolate RNA, which was analyzed by quantitative reverse-transcription polymerase chain reaction to assess the effect of verinurad on CYP1A2, CYP2B6, and CYP3A4 mRNA levels.

Cells and Culture Conditions. Cryopreserved hepatocytes for hepatic uptake studies were purchased from Triangle Research Laboratories (Research Triangle Park, NC). Drug transporter interaction studies were performed using cell lines or vesicles with either endogenous (Caco-2), stable, or transient expression of the transporter, and corresponding background control systems (empty vector-transfected cells). Origins of all in vitro systems are summarized in Supplemental Tables 4 and 5 . The culture conditions followed established and validated protocols (Zhang et al., 2015; Yu et al., 2016; Fredlund et al., 2017).

Determination of Intrinsic Permeability of Verinurad. Intrinsic permeability of verinurad was tested at a concentration of 10 $\mu \mathrm{M}$ in Caco-2 cell monolayers, as previously described (Fredlund et al., 2017). Cells were cultivated for 2 weeks in Transwell filter plates (Corning Corporation, $0.4 \mu \mathrm{m}$ pore size), and the study was performed in the apical-basolateral direction with a $\mathrm{pH}$ gradient of 6.5/7.4, reflecting the slightly acidic upper intestinal $\mathrm{pH}$ in the donor compartment. Samples from the receiver compartment were collected over a time interval of 120 minutes and analyzed with LC-MS/MS.

Determination of Passive and Active Hepatic Uptake of Verinurad in Cryopreserved Human Hepatocytes. The assay was conducted by QPS LLC (Newark, DE) as described previously (Kimoto et al., 2011, 2019). The hepatic uptake mechanism of verinurad was investigated at 0.5 and $5 \mu \mathrm{M}$ in suspended cryopreserved human hepatocytes (final concentration of $1 \times 10^{6}$ cells $/ \mathrm{ml}$ ). Hepatocytes were preincubated with a mixture of $25 \mu \mathrm{M}$ rifamycin $\mathrm{SV}$ and $25 \mu \mathrm{M}$ prazosin (inhibitors of OATPs and OCT1) or buffer/solvent control at $37^{\circ} \mathrm{C}$ for 10 minutes. Uptake was initiated by the addition of verinurad as the test compound or positive control substrate (rosuvastatin for active uptake and verapamil for passive uptake). At time points $0.5,1$, and 1.5 minutes, samples were transferred to a centrifuge tube containing a mix of mineral and silicone oil over a bottom layer of $2 \mathrm{M}$ ammonium acetate. The tubes were immediately centrifuged for 30 seconds at $18,300 \mathrm{~g}$ to separate cells from the buffer and subsequently frozen down. Each tube was cut near the oil/base interface, and the cell pellet was resuspended in $50 \mu \mathrm{l}$ water and $100 \mu \mathrm{l}$ of the appropriate internal standard in acetonitrile. The samples were vortexed for 6 minutes at high speed to extract the pellet, which was followed by centrifugation at $3000 \mathrm{rpm}$ for 10 minutes. Substrate concentration in the supernatant was determined using LC-MS/MS (Supplemental Table 1). The percent active uptake was quantified from the slope of uptake in the absence and presence of inhibitors. Each treatment group was conducted in quadruplicate.

Evaluation of Verinurad, M1, and M8 as Substrates and Inhibitors of Human Drug Transporters. Assays were conducted by Optivia Biotechnology (Menlo Park, CA), QPS LLC, Pharmaron (Beijing, China), and Cyprotex Discovery Ltd (Macclesfield, UK). The in vitro models used to study the transporter interactions are summarized in Table 1. 
TABLE 1

Summary of in vitro test systems and assays

\begin{tabular}{|c|c|c|c|}
\hline & Transporter & Test System & Assay \\
\hline \multicolumn{4}{|c|}{ Verinurad Transporter Substrate Assays } \\
\hline \multirow[t]{3}{*}{ Efflux } & P-gp & Transfected MDCKII cells & Transcellular transport \\
\hline & BCRP & & Transcellular transport \\
\hline & MRP2, MRP4 $4^{\mathrm{a}}$ & Membrane vesicles & Uptake \\
\hline \multirow[t]{2}{*}{ Hepatic } & OATP1B1, OATP1B3, OCT1 & Transfected MDCKII cells & Uptake \\
\hline & OATP2B1 & CHO cells & Uptake \\
\hline Renal & OCT2, OAT1, OAT3, MATE1, MATE2-K & Transfected MDCKII cells & Uptake \\
\hline & BCRP & Caco-2 cells & Transcellular transport \\
\hline & $\mathrm{MRP}^{\mathrm{b}}, \mathrm{MRP}^{\mathrm{b}}, \mathrm{BSEP}^{\mathrm{c}}$ & Membrane vesicles & Uptake \\
\hline Hepatic & OATP1B1, OATP1B3, OAT2 ${ }^{\mathrm{c}}$, OCT1 & $\begin{array}{c}\text { Transfected MDCKII or } \\
\text { HEK-293 cells }\end{array}$ & Uptake \\
\hline Renal & OCT2, OAT1, OAT3, MATE1 ${ }^{\mathrm{b}}$, MATE2-K ${ }^{\mathrm{b}}$ & $\begin{array}{c}\text { Transfected MDCKII or } \\
\text { HEK-293 cells }\end{array}$ & Uptake \\
\hline
\end{tabular}

${ }^{\text {a }}$ Metabolites M1 and M8 were also assessed as substrates of MRP2 and MRP4.

${ }^{\mathrm{b}} \mathrm{M} 1$ and M8 only.

${ }^{\mathrm{c}}$ Verinurad only.

The methods for the substrate and inhibition assays have been described previously (Zhang et al., 2015; Yu et al., 2016). Furthermore, Supplemental Tables 4 and 5 summarize the experimental conditions used to characterize the substrate and inhibitory interaction of verinurad, M1, and M8 with human drug transporters. Transporter substrate studies included control systems and specific inhibitors to corroborate the significance of the observed in vitro substrate data. Transporter inhibition studies were conducted with index substrates at multiple concentrations of test drug (verinurad, M1, or M8) over a clinically relevant concentration range. All reactions were performed in triplicate.

Determination of Verinurad as a Substrate of P-Glycoprotein and Breast Cancer Resistance Protein in Madin-Darby Canine Kidney II-MDR1 and Caco-2 Cells. Madin-Darby Canine Kidney (MDCK) II-MDR1 and Caco-2 cells were grown to confluency in 24-well Transwell plates, washed in assay buffer, and preincubated at $37^{\circ} \mathrm{C}$ for $20-30$ minutes. Verinurad or ${ }^{14} \mathrm{C}$-verinurad was either added to the apical or basolateral side and incubated with orbital shaking at 50-60 rpm. The appearance of verinurad in the opposite receiver chamber was measured for 120 minutes. ${ }^{14} \mathrm{C}$-Verinurad was quantified with radiometric detection on a 1450 Microbeta (PerkinElmer, CT), whereas unlabeled verinurad was quantified with LCMS/MS (Supplemental Table 1).

Determination of Verinurad as a Substrate of Human Solute Carrier Transporters. The potential for verinurad to be a substrate of human drug transporters OCT1, OCT2, OAT1, OAT3, MATE1, MATE2-K, OATP1B1, and OATP1B3 was evaluated in MDCKII cells transiently transfected with the respective drug transporter of interest. OATP2B1 interaction was studied in OATP2B1transfected Chinese hamster ovary (CHO) cells.

Cellular uptake of ${ }^{14} \mathrm{C}$-verinurad was studied over 5 minutes of incubation at $37^{\circ} \mathrm{C}$ with orbital shaking at 50-60 rpm. Then, cells were quickly rinsed with an ice-cold phosphate-buffered solution, and a cell extraction solution (50:50 mixture of acetonitrile and water) was added. The intracellular ${ }^{14} \mathrm{C}$-verinurad concentration was quantified with radiometric detection.

Determination of Verinurad, M1, and M8 as Substrates of Efflux Transporters Multidrug Resistance-Associated Proteins 2 and 4. Studies of verinurad and metabolites M1 and M8 as substrates of efflux transporters multidrug resistance-associated protein (MRP) 2 and MRP4 were performed by Optivia Biotechnology using Spodoptera frugiperda (Sf9) insect vesicles expressing human MRP2 or MRP4. Briefly, samples were incubated with blocking buffer (40 mM MOPS-Tris $\mathrm{pH} 7.0,70 \mathrm{mM}$ potassium chloride $(\mathrm{KCl})$, and 0.5 $\mathrm{mg} / \mathrm{ml}$ bovine serum albumin) at $37^{\circ} \mathrm{C}$ for 60 minutes with orbital shaking at $200 \mathrm{rpm}$. Blocking buffer was removed, and assay uptake buffer (50 mM MOPS-Tris pH 7.0, $70 \mathrm{mM} \mathrm{KCl,} 7 \mathrm{mM}$ magnesium chloride, and $3 \mathrm{mM}$ L-glutathione reduced) containing MRP2 or MRP4 vesicles was added. Verinurad, M1, M8, or positive control substrate with and without reference inhibitor were added and preincubated at $37^{\circ} \mathrm{C}$ with orbital shaking at $200 \mathrm{rpm}$ for 10 minutes. To start uptake, adenosine triphosphate (ATP) or adenosine monophosphate (AMP) was added to the plates to a final concentration of $5 \mathrm{mM}$, and the plates were incubated for 5 (MRP2) or 10 minutes (MRP4). Incubations were stopped by the addition of an ice-cold wash buffer (50 mM MOPS-Tris $\mathrm{pH} 7.0$ and $70 \mathrm{mM} \mathrm{KCl}$ ). The samples were transferred to glass fiber filtration plates to separate vesicles from the buffer by applying a vacuum. The filter plate wells were washed and allowed to dry under vacuum. Filter wells were punched out, and radiolabeled substrate was quantified with radiometric detection on a 1450 Microbeta. Unlabeled M1 and M8 were quantified by LC-MS/MS (Supplemental Table 1).

Determination of Verinurad, M1, and M8 as Inhibitors of Human Efflux Transporters P-Glycoprotein and Breast Cancer Resistance Protein. The potential for verinurad, M1, and M8 to inhibit the transport of probe substrates by human efflux transporters P-glycoprotein (P-gp) and breast cancer resistance protein (BCRP) was performed using MDCKII-MDR1 or Caco-2 cells by Optivia Biotechnology or Pharmaron. Essential assay details are summarized in Supplemental Table 5. Radiolabeled substrates were analyzed using liquid scintillation counting (Microbeta), whereas other substrates were quantified using LC-MS/MS (Supplemental Table 1).

Determination of Verinurad, M1, and M8 as Inhibitors of Human Solute Carrier Transporters. Human solute carrier transporter inhibitor studies were performed by Optivia Biotechnology, Pharmaron, and Cyprotex Discovery Ltd. The potential for verinurad, M1, and M8 to inhibit the transport of established probe substrates for human uptake transporters (OATP1B1, OATP1B3, OAT1, OAT2, OAT3, OCT2, and OCT1) and apical efflux transporters (MATE1 and MATE2-K) was determined as summarized in Supplemental Table 5. Reference inhibitors were tested in parallel to qualify the test system and ensure that the results met the acceptance criteria. Cells were preincubated at $37^{\circ} \mathrm{C}$ with orbital shaking for the preincubation times specified in Supplemental Table 5. Then, probe substrate and a concentration series of test compound or positive control inhibitor were added and incubated at $37^{\circ} \mathrm{C}$ with orbital shaking for the incubation times specified in Supplemental Table 5. Uptake was stopped by removing the incubation solutions and rinsing the cell monolayers with ice-cold Hanks' balanced salt solution buffer. Cells were lysed for at least 5 minutes at $250 \mathrm{rpm}$ on an orbital shaker, and probe substrate concentrations were determined by LC-MS/MS or scintillation counting. 
Determination of Verinurad, M1, and M8 as Inhibitors of Human Efflux Transporters Bile Salt Export Pump, MRP2, and MRP4. The potential for verinurad and metabolites M1 and M8 to inhibit the transport of probe substrates by human efflux transporters bile salt export pump (BSEP), MRP2, and MRP4 was determined using Sf9 vesicles expressing the respective transporter (Supplemental Table 5). The methods used were similar to those described for the substrate transport assays using Sf9 inverted membrane vesicles. Membrane vesicles were preincubated with the probe substrate and a concentration series of test compound or positive control inhibitor at $37^{\circ} \mathrm{C}$ with orbital shaking for 10 minutes. To start the uptake process, ATP or AMP was added to a final concentration of $5 \mathrm{mM}$, and the samples were incubated at $37^{\circ} \mathrm{C}$ with orbital shaking for 5 minutes for MRP2, 10 minutes for MRP4, and 15 minutes for BSEP. Uptake reactions were stopped by fast filtration using a glass fiber filtration plate and washing with wash buffer. The amount of substrate in the filters was quantified either by LC-MS/MS or with radiometric detection.

LC-MS/MS Analysis. LC-MS/MS analysis of verinurad, M1, M8, probe substrates (transporter assays), and enzyme-specific metabolites (enzyme inhibition and induction assays) varied according to the service contractor used. The system conditions used for sample analysis (e.g., type of column, flow rate, gradient profile, and analysis time) and transitions monitored for the analytes are summarized in Supplemental Table 1.

LC-MS/MS analysis of verinurad, M1, and M8 in the in vitro assays was based on the methods described previously (Lee et al., 2018), wherein $\mathrm{D}_{6}$ internal standards of each analyte were available for analysis.

Data Analysis. For assays run in triplicate, mean and standard deviation are reported.

In Vitro Plasma Protein Binding in Human (Free Fraction). For the determination of the PPB of M1 and M8, the percentage of unbound drug metabolite was calculated as:

$$
\% \text { Unbound }=\frac{\text { Concentration }_{\text {filtrate }}}{\text { Concentration }_{\text {total }}} \times 100
$$

Enzyme Inhibition IC $_{50}$ Calculations (CYP and UGT). Inhibition of metabolizing enzyme activity was expressed as the percentage decrease in the activity of a marker metabolite formation compared with noninhibited controls (100\% activity). Mean enzyme activity $(n=3)$ for each test concentration was plotted against the log inhibitor concentration and fitted to an $\mathrm{IC}_{50}$ curve using GraphPad Prism or Excel XLfit (5.3.1.3):

$$
Y=\text { Bottom }+\frac{\text { Top }- \text { Bottom }}{1+10^{\left(\log I C_{50}-X\right)}}
$$

in which $\mathrm{X}$ is the logarithm of inhibitor concentration, $\mathrm{Y}$ is the percent of control activity and $\mathrm{Y}$ starts at the bottom and goes to the top with a sigmoid shape, and Top and Bottom are the $\mathrm{Y}$ values at the top and bottom plateau of the curve.

No inhibition (NI) represents no inhibition at the highest concentration tested, and $\mathrm{IC}_{50}>30$ or $>100 \mu \mathrm{M}$ (for CYPs with metabolites and verinurad, respectively) and $>1250 \mu \mathrm{M}$ (for UGTs) represent inhibition at the highest concentration tested but not enough to obtain an accurate $\mathrm{IC}_{50}$ determination.

The TDI of each CYP450 enzyme in HLMs was calculated as percent NADPH-dependent activity shift after preincubation in the absence and presence of CYP-cofactor NADPH as follows:

$$
\% T D I=\left(1-\frac{\% \text { control activity }{ }^{N A D P H}}{\% \text { control activity }{ }^{N o N A D H}}\right) \times 100
$$

Transporter Substrate Determinations. In bidirectional transport assays, efflux transporter substrates were characterized through efflux ratios (ERs) (Supplemental Table 4).

Permeability of reference substrate or test drug was estimated as apparent permeability $\left(\mathrm{P}_{\mathrm{app}}\right)$ :

$$
P_{a p p}=\frac{1}{\text { Area } \times C_{D}(0)} \times \frac{d M_{r}}{d t}(\mathrm{~cm} / s)
$$

in which Area is the area of the filter $\left(0.33 \mathrm{~cm}^{2}\right), \mathrm{C}_{\mathrm{D}}(0)$ is the initial substrate concentration in the donor compartment, and $\mathrm{dM}_{\mathrm{r}} / \mathrm{dt}$ is the flux rate of test drug to the receiver compartment.

ER was expressed as a ratio of $\mathrm{B} \rightarrow \mathrm{A}$ over $\mathrm{A} \rightarrow \mathrm{B} \mathrm{P}_{\text {app}}$ :

$$
E R=\frac{P_{\mathrm{app}}(B \rightarrow A)}{P_{\mathrm{app}}(A \rightarrow B)}(\text { dimensionless })
$$

In the human uptake transporter substrate assays, uptake in the test system (expressed the transporter of interest) was compared with uptake in the control system (did not express the transporter of interest) using unpaired Student's $t$ test with Holm-Sidak correction for multiple comparisons. A $P$ value of $<0.05$ was considered indicative of active transporter-mediated uptake of the test article. Verification of transporter specificity in the human uptake transporter substrate assays was performed by adding a strong reference inhibitor. Percent inhibition by the reference inhibitor was calculated by:

$\%$ inhibition $=$

$$
\left(1-\frac{(\text { Net transporter }- \text { mediated uptake })_{\text {with inhibitor }}}{(\text { mean Net transporter }- \text { mediated uptake })_{\text {without inhibitor }}}\right) \times 100
$$

in which net transporter-mediated uptake was calculated by subtracting uptake in the control system from uptake in the test system.

IC $_{50}$ Calculations for Transporters. To determine the $\mathrm{IC}_{50}$ of verinurad or its metabolites in uptake and efflux experiments, data were fitted by nonlinear regression to a sigmoidal variable slope $\mathrm{IC}_{50}$ model (GraphPad Prism or Excel XLfit [5.3.1.3, Eq. 201]):

$$
Y=\text { Bottom }+\frac{\text { Top-Bottom }}{1+\left(\frac{I C_{50}}{X}\right)^{n}}
$$

in which $\mathrm{Y}$ is the percent control activity/percent inhibition in the presence of the test article throughout the concentration range tested, Top is the percent control activity/percent inhibition in the absence of the test article, $\mathrm{X}$ is the inhibitor concentration, and $n$ is a Hill coefficient. Bottom can be fixed to 0 when defined through background experiments with full inhibition.

To determine the $\mathrm{IC}_{50}$ in transcellular flux experiments (i.e., MDCKII-MDR1 and Caco-2 [BCRP] inhibition assays), percent control activity values were calculated using the below equation:

$$
\% \text { control activity }=\frac{P_{a p p(+ \text { test compound })}-P_{\text {app }(\text { passive })}}{P_{\text {app }(\text { vehicle control })}-P_{\text {app }(\text { passive })}} \times 100
$$

in which $\mathrm{P}_{\mathrm{app} \text { (+ test compound) }}$ is the $\mathrm{P}_{\mathrm{app}}$ of substrate in the presence of test compound, $\mathrm{P}_{\text {app(vehicle control) }}$ is the mean $\mathrm{P}_{\text {app }}$ of substrate in the absence of test compound, and $\mathrm{P}_{\text {app(passive) }}$ is the mean $\mathrm{P}_{\mathrm{app}}$ of substrate in the presence of the highest concentration of positive control inhibitor assuming 100\% inhibition at this concentration. From this, percent control activity values in the presence of a range of test compound/inhibitor concentrations were plotted against nominal inhibitor concentration and fitted to the 4 Parameter Logistic model above.

To determine the $\mathrm{IC}_{50}$ in intracellular accumulation assays, the percent inhibition of the net transporter-mediated uptake was calculated according to eq. 6 and plotted against nominal inhibitor 
concentrations. $\mathrm{IC}_{50}$ values were subsequently calculated by fitting the data to eq. 7.

Risk Assessment and Margin Calculations for Transporter and Enzyme Inhibition. As recommended by regulatory agencies (European Medicines Agency, 2013; Japanese Pharmaceuticals and Medical Devices Agency, 2018; Food and Drug Administration, 2020), the degree of inhibition in humans was estimated by relating the observed $\mathrm{IC}_{50}$ to maximal total $\left(\mathrm{I}_{\max }\right.$ or $\mathrm{C}_{\max }$ ) or free drug concentration $\left(\mathrm{I}_{\mathrm{u}, \max }\right.$ or $\mathrm{C}_{\mathrm{u} \text {,max }}$ ) (Table 2) and/or by calculating $\mathrm{R}$ values as defined by the US FDA 2020 guidance. The combined inhibitory potential of verinurad and its metabolites was calculated as a sum of the individual ratios of exposure in relation to in vitro inhibition constants.

The anticipated worst case therapeutic $\mathrm{C}_{\max }$ values of verinurad, M1, and M8 were $0.238 \mu \mathrm{M}, 0.416 \mu \mathrm{M}$, and $0.409 \mu \mathrm{M}$, respectively, based on the highest dose of verinurad (i.e., $24 \mathrm{mg}$ ) currently being tested in phase 2 studies (NCT03990363 and NCT04327024). The $\mathrm{C}_{\max }$ of verinurad was estimated from the observed $\mathrm{C}_{\max }(36.3 \mathrm{ng} / \mathrm{ml}$ or $0.104 \mu \mathrm{M})$ in healthy volunteers receiving verinurad $12 \mathrm{mg}$ once daily (unpublished AstraZeneca data on file), adjusting for differences in dose and accounting for the 2.28-fold higher exposure seen in those with severe renal impairment after a single dose of verinurad (Smith et al., 2018).

The $\mathrm{C}_{\max }$ values of $\mathrm{M} 1$ and $\mathrm{M} 8$ were estimated from observed $\mathrm{C}_{\max }$ values (91.0 and $87.2 \mathrm{ng} / \mathrm{ml}$, respectively, or 0.173 and $0.161 \mu \mathrm{M}$, respectively) in subjects with renal impairment receiving a single dose of $15 \mathrm{mg}$ verinurad, accounting for the difference in dose, relative bioavailability between formulations (60\%), and the expected accumulation ratio of M1 and M8 (using a terminal half-life of 21.2 and 22.8 hours, respectively) after $12 \mathrm{mg}$ once daily verinurad dosing (Smith et al., 2018).

\section{Results}

Permeability of Verinurad. The intrinsic permeability of verinurad in Caco-2 cells was $35.8 \times 10^{-6} \mathrm{~cm} / \mathrm{s}$, with a $\mathrm{P}_{\mathrm{app}}$ value higher than that for the high-permeability reference compound metoprolol $\left(18.3 \times 10^{-6} \mathrm{~cm} / \mathrm{s}\right)$.

Plasma Protein Binding of Verinurad, M1, and M8. For the equilibrium dialysis assessment of verinurad, the average ratio in the drug chamber and sample chamber was $0.993 \pm 0.008$, indicating equilibrium was reached using the rapid equilibrium dialysis device. In human plasma, verinurad was highly bound (average $97.1 \%$ ) to plasma protein between 1 and $50 \mu \mathrm{M}$ with minimal differences observed with increasing concentration (Table 3). Binding of verinurad to HSA was $>96.3 \%$ at all concentrations evaluated and was similar to the fraction bound in human plasma (Table 3). The extent of binding to AGP was $<10 \%$ at all concentrations, indicating that binding in human plasma is primarily to serum albumin (Table 3).

Because of the difference in stability of the acyl glucuronide metabolites compared with the parent drug, the PPB of metabolites M1 and M8 was determined by ultracentrifugation. The protein binding experiments using ultracentrifugation showed that both M1 and M8 had good stability (>85\%) over 60 minutes. The mean percent recovery of M1 was $93.6 \pm$ $3.9 \%$ and $95.1 \pm 3.92 \%$ at 1 and $50 \mu \mathrm{M}$, respectively, and for M8 was $86.6 \pm 3.4 \%$ and $85.9 \pm 2.2 \%$ at 1 and $50 \mu \mathrm{M}$, respectively. Our results showed that M1 was highly protein bound (average $92.1 \%$ ), and M8 was moderately protein bound (average $66.2 \%$ ) in human plasma at 1 and $50 \mu \mathrm{M}$ (Table 3).

Passive and Active Hepatic Uptake of Verinurad. The mechanism of hepatic uptake of verinurad was determined based on the rate of its appearance in suspended human hepatocytes in the absence and presence of a mixture of $25 \mu \mathrm{M}$ prazosin and $25 \mu \mathrm{M}$ rifamycin SV (inhibitors of the major hepatic uptake transporters OCT1 and OATPs,

TABLE 2

Equations for risk assessments/margin calculations for transporter and enzyme inhibition as described by the EMA (2013), US FDA (2020), or JPMDA (2018)

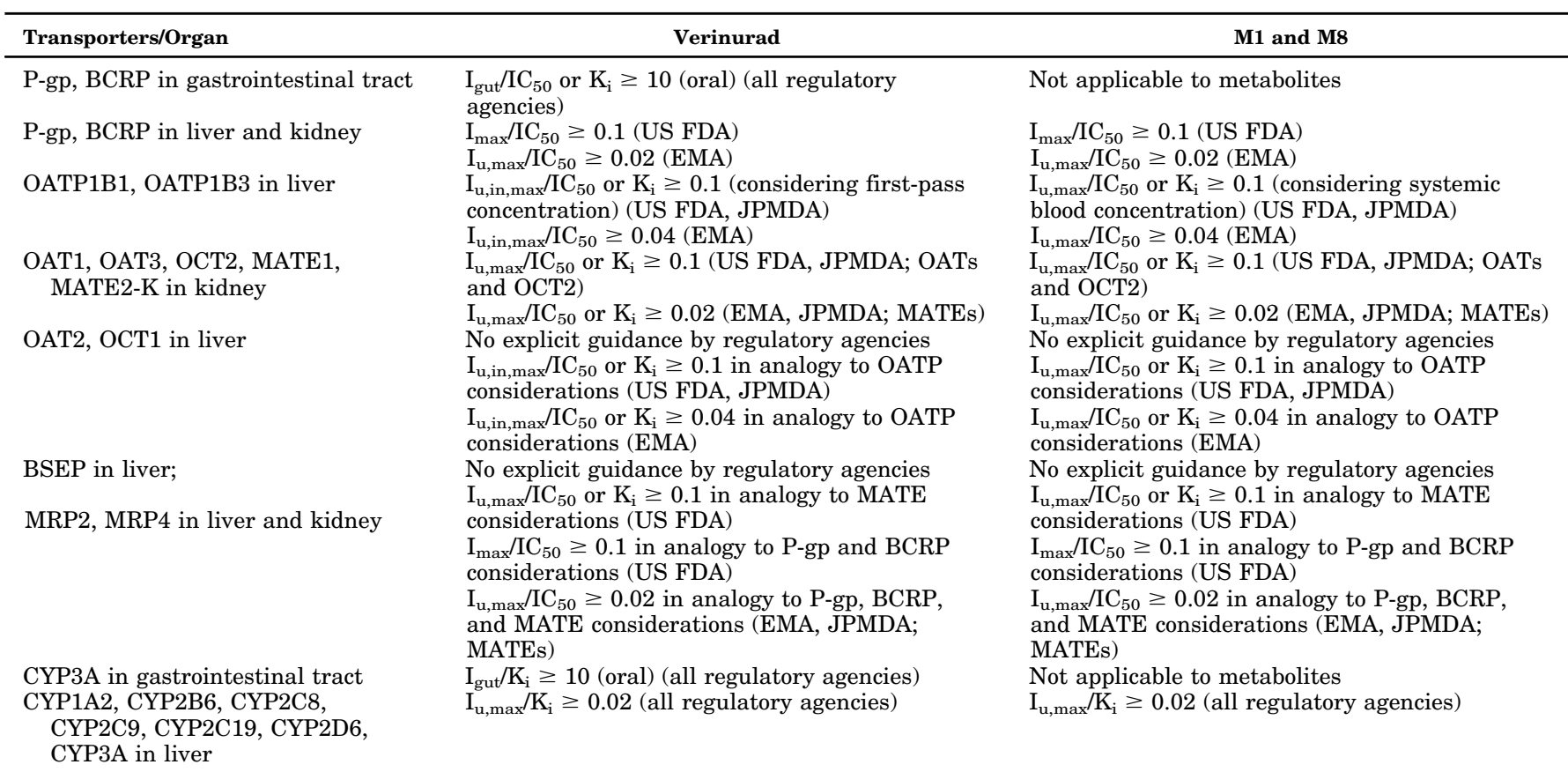

EMA, European Medicines Agency; FDA, Food and Drug Administration; $\mathrm{f}_{\mathrm{u}}$, unbound fraction in plasma; $\mathrm{I}_{\mathrm{gut}}$, intestinal luminal concentration; $\mathrm{I}_{\mathrm{max}}$, total maximum plasma inhibitor concentration; $I_{u, \text { in, max }}$, maximum free hepatic inlet concentration; $I_{u, \text { max }}$, maximum free plasma concentration; JPMDA, Japanese Pharmaceuticals and Medical Devices Agency; $\mathrm{K}_{\mathrm{i}}$, inhibitory constant. 
TABLE 3

Protein binding of verinurad and metabolites M1 and M8 in human plasma, HSA, and AGP

\begin{tabular}{llccc}
\hline & & \multicolumn{3}{c}{$\%$ Protein Binding $($ Mean \pm S.D., $\mathbf{N}=\mathbf{3})$} \\
\cline { 3 - 5 } Test Compound & & $\mathbf{1} \mu \mathbf{M}$ & $\mathbf{1 0} \mu \mathbf{M}$ & $\mathbf{5 0} \mu \mathbf{M}$ \\
\hline Verinurad & Plasma & $97.4 \pm 0.3$ & $97.3 \pm 0.1$ & $96.7 \pm 0.1$ \\
& HSA & $97.3 \pm 0.3$ & $96.8 \pm 0.3$ & $96.3 \pm 0.1$ \\
M1 & AGP & $9.8 \pm 0.9$ & $8.4 \pm 2.5$ & $8.6 \pm 1.7$ \\
M8 & Plasma & $92.4 \pm 0.21$ & - & $91.6 \pm 0.28$ \\
& Plasma & $65.2 \pm 0.23$ & - & $67.2 \pm 2.17$ \\
\hline
\end{tabular}

AGP, $\alpha$-1-acid glycoprotein; HSA, human serum albumin; SD, standard deviation

respectively). The uptake profiles of verinurad with and without transporter inhibitors were different, indicating an active uptake mechanism similar to that of active control substrate rosuvastatin (Supplemental Table 6). The calculated percent active uptake of verinurad was $51 \%$ at $0.5 \mu \mathrm{M}$ and $86 \%$ at 5 $\mu \mathrm{M}$, indicating that hepatic uptake of verinurad involves both passive and active mechanisms. For the passive control substrate verapamil, there was no difference in the uptake slope with and without inhibitor (Supplemental Table 6).

In Vitro Evaluation of Verinurad, M1, and M8 as Inhibitors of Human Metabolizing Enzymes. From the panel of CYP enzymes and over the verinurad concentrations tested $(0.016-100 \mu \mathrm{M})$, verinurad inhibited CYP2C8, CYP2C9, and CYP3A4/5, with $\mathrm{IC}_{50}$ values of $14.5 \mu \mathrm{M}, 25.6 \mu \mathrm{M}$, and 77.1/85.3 $\mu \mathrm{M}$, respectively (Table 4). For the other CYPs examined, $<50 \%$ inhibition at the highest concentration tested was observed, with $\mathrm{IC}_{50}$ values determined to be $>100 \mu \mathrm{M}$ (Table 4). The $\mathrm{IC}_{50}$ values of verinurad as an inhibitor of UGT1A1 and UGT2B7 were $192.7 \mu \mathrm{M}$ and $1120 \mu \mathrm{M}$, respectively (Table 4). For M1, although inhibition of CYP1A2, CYP2B6, and CYP2C8 activity was observed at the highest concentration tested, the inhibition was insufficient to accurately determine $\mathrm{IC}_{50}$ values $\left(\mathrm{IC}_{50}>30 \mu \mathrm{M}\right.$; Table 4$)$. Likewise, $<20 \%$ inhibition of CYP1A2, CYP2A6, CYP2B6, and CYP2E1 activity by M8 was observed at the highest concentration tested, with $\mathrm{IC}_{50}$ values $>30 \mu \mathrm{M}$ (Table 4). There was no evidence that M1 or M8 inhibited other CYP enzymes studied over the concentration range tested (0.1-30.0 $\mu \mathrm{M}$; Table 4).

Preincubation with verinurad, M1, and M8 did not enhance inhibitory potency toward any CYP enzyme studied (CYP1A2, 2C8, 2C9, 2C19, 2D6, and 3A4/5); either $<20 \%$ TDI was observed at the highest test concentration, or $\mathrm{IC}_{50}$ values did not decrease upon preincubation. By contrast, preincubation of HLMs with reference inhibitors for CYP1A2 $(10 \mu \mathrm{M}$ furafylline), CYP2C8 (10 $\mu \mathrm{M}$ gemfibrozil-1- $O-\beta$ glucuronide), CYP2C9 (2.5 $\mu \mathrm{M}$ tienilic acid), CYP2C19 (5 $\mu \mathrm{M}$ ticlopidine), CYP2D6 (5 $\mu \mathrm{M}$ paroxetine), and CYP3A4/5 (1.5 $\mu \mathrm{M}$ troleandomycin) resulted in TDI as expected. This indicates that verinurad, $\mathrm{M} 1$, and M8 are not time-dependent inhibitors of CYP1A2, CYP2C8, CYP2C9, CYP2C19, CYP2D6, and CYP3A4/5.

In Vitro Evaluation of Verinurad as a CYP Inducer in Human Metabolizing Enzymes. Treatment of cultured hepatocytes with up to $100 \mu \mathrm{M}$ verinurad had less than 2-fold change on CYP1A2, CYP2B6, and CYP3A4 mRNA expression levels (Supplemental Table 7). There was a trend for a concentration-dependent increase in CYP3A4/5 activity in all three hepatocyte cultures with verinurad; however, verinurad was less than $20 \%$ (13.5\% on average) as effective as rifampicin at inducing CYP3A4/
5 activity at the highest concentration tested in all donors (Supplemental Table 8). This is below the 20\% threshold from the US FDA (Food and Drug Administration, 2020) and collectively indicates low risk of induction.

In Vitro Evaluation of Verinurad, M1, and M8 as Substrates of Human Drug Transporters. Verinurad and its metabolites M1 and M8 were evaluated as substrates of drug transporters in various in vitro test systems (Supplemental Table 4). Functional transport activity in each test system was confirmed by transport studies using probe substrates and specific inhibitors. The resulting findings are summarized in Figs. 2 and 3 (efflux transporters), Fig. 4 (renal transporters), and Fig. 5 (hepatic uptake transporters).

Transport of Verinurad, M1, and M8 by Ubiquitously Expressed Efflux Transporters P-gp, BCRP, MRP2, and MRP4. In vitro, verinurad was a substrate of Pgp, with an ER $>2$ at 1 and $10 \mu \mathrm{M}$ in MDCKII-MDR1 cells. The efflux was inhibited by the reference inhibitor ketoconazole, whereas verapamil did not reduce the ER of $10 \mu \mathrm{M}$ verinurad to $<2$, indicating a potential involvement of other efflux transporters alongside P-gp (Fig. 2A). In additional studies in MDCKII-MDR1 cells, verinurad had ERs of 1.80-2.53 at concentrations of 1-30 $\mu \mathrm{M}$ (Fig. 2B). The addition of the reference inhibitor elacridar decreased the ER of verinurad to $<1$ at 1 $\mu \mathrm{M}$ and $3 \mu \mathrm{M}$, and $<1.5$ at $10 \mu \mathrm{M}$ and $30 \mu \mathrm{M}$ (Fig. 2B), indicating that verinurad is a substrate of P-gp in vitro.

Verinurad was also identified as a substrate of BCRP, with an ER $>2$ at concentrations of $0.3,1$, and $5 \mu \mathrm{M}$ in Caco-2 cells; however, the ER remained $>2$ at $5 \mu \mathrm{M}$ verinurad after the addition of the reference inhibitor chrysin (Fig. 2C). In a follow-up experiment in MDCKII-BCRP cells, the ER of verinurad was $>2$ at concentrations of 0.5 and $2 \mu \mathrm{M}$ (Fig. 2D). The ER was reduced to $<2$ at both concentrations after the addition of reference inhibitors KO143 and elacridar (Fig. 2D), suggesting that verinurad is a substrate for human BCRP in vitro.

The evaluation of verinurad and metabolites M1 and M8 as substrates of MRP2 and MRP4 was conducted in Sf9 insect vesicles expressing human MRP2 or MRP4. ATP did not significantly increase uptake of verinurad compared with AMP controls $(P>0.05)$ in neither MRP2 (Fig. 3A) nor MRP4 vesicles (Fig. 3B), indicating that verinurad is not a substrate of MRP2 or MRP4 in vitro. In contrast, both M1 and M8 demonstrated significantly higher uptake in MRP2 vesicles in the presence of ATP compared with AMP (M1: $P=0.0001$; M8: $P<0.0001$ ) (Fig. 3, C and D). The reference inhibitor benzbromarone clearly inhibited MRP2-mediated uptake of $1 \mu \mathrm{M}$ of $\mathrm{M} 1$ by $99.7 \%$ and $1 \mu \mathrm{M}$ of M8 by $99.2 \%$ (Fig. 3, C and D), confirming that they are substrates of MRP2 in vitro. Similarly, both M1 and M8 demonstrated concentration-dependent uptake in MRP4 vesicles in the presence of ATP compared with AMP (M1: $P \leq 0.0002$; M8: $P=0.0124$ ) (Fig. $3, \mathrm{E}$ and F). The MRP4-mediated uptake of $1 \mu \mathrm{M}$ of M1 and $1 \mu \mathrm{M}$ of M8 was inhibited by benzbromarone by $91.5 \%$ and $100.0 \%$, respectively (Fig. 3, E and F), confirming that they are substrates of MRP4 in vitro.

Transport of Verinurad by Human Renal Transporters. In vitro uptake studies showed OAT1-dependent uptake of verinurad in MDCKII-OAT1 cells at concentrations of $0.1-10 \mu \mathrm{M}$, with statistically significant differences between MDCKII-OAT1 and control cells at the four highest concentrations $(P<0.05)$ (Fig. 4A), indicating that verinurad is a 
TABLE 4

In vitro evaluation of direct inhibition of metabolizing enzymes by verinurad and metabolites M1 and M8R. $R, R_{1}$, and $R_{1, \text { gut }}$ calculations follow the US FDA 2020 guidance

\begin{tabular}{|c|c|c|c|c|c|}
\hline Enzyme & $\mathrm{IC}_{50}(\boldsymbol{\mu} \mathrm{M})$ & $\mathbf{I}_{\mathbf{u}, \max } / \mathbf{I C}_{50}$ & $\mathbf{R}$ & $\mathbf{R}_{1}$ & $\mathbf{R}_{1, \text { gut }}$ \\
\hline \multicolumn{6}{|l|}{ CYP1A2 } \\
\hline Verinurad & $>100$ & _- & _- & - & - \\
\hline M1 & $>30$ & - & - & - & - \\
\hline M8 & $>30$ & - & _- & - & - \\
\hline \multicolumn{6}{|l|}{ CYP2A6 } \\
\hline Verinurad & - & - & - & - & - \\
\hline M1 & NI & _- & _ & - & - \\
\hline M8 & $>30$ & - & - & - & - \\
\hline \multicolumn{6}{|l|}{ CYP2B6 } \\
\hline Verinurad & $>100$ & _- & _- & - & - \\
\hline M1 & $>30$ & - & - & - & - \\
\hline M8 & $>30$ & - & _- & - & - \\
\hline \multicolumn{6}{|l|}{ CYP2C8 } \\
\hline Verinurad & 14.5 & 0.00049 & - & 1.00049 & - \\
\hline M1 & $>30$ & - & _- & - & - \\
\hline M8 & NI & - & - & - & - \\
\hline \multicolumn{6}{|l|}{ CYP2C9 } \\
\hline Verinurad & 25.6 & 0.00028 & 1.00028 & 1.00028 & - \\
\hline M1 & $\mathrm{NI}$ & - & - & - & - \\
\hline M8 & NI & - & _- & - & - \\
\hline \multicolumn{6}{|l|}{ CYP2C19 } \\
\hline Verinurad & $>100$ & - & - & - & - \\
\hline M1 & NI & - & _- & - & - \\
\hline M8 & NI & - & _- & - & - \\
\hline \multicolumn{6}{|l|}{ CYP2D6 } \\
\hline Verinurad & $>100$ & - & _- & - & - \\
\hline M1 & NI & _- & _- & _- & - \\
\hline M8 & NI & - & - & - & - \\
\hline \multicolumn{6}{|l|}{ CYP3A4/5 } \\
\hline Verinurad & 77.1 and $85.3^{\mathrm{a}}$ & 0.00009 & _ & 1.00009 & 4.6 \\
\hline M1 & NI & - & - & - & - \\
\hline M8 & NI & - & _- & - & - \\
\hline \multicolumn{6}{|l|}{ CYP2E1 } \\
\hline Verinurad & - & - & - & - & - \\
\hline M1 & NI & - & _- & - & - \\
\hline M8 & $>30$ & - & - & - & - \\
\hline \multicolumn{6}{|l|}{ UGT1A1 } \\
\hline Verinurad & 192.7 & _- & _- & - & - \\
\hline M1 & - & - & - & - & - \\
\hline M8 & - & - & - & - & - \\
\hline \multicolumn{6}{|l|}{ UGT2B7 } \\
\hline Verinurad & 1120 & - & - & - & - \\
\hline M1 & - & - & - & - & - \\
\hline M8 & - & - & - & - & - \\
\hline
\end{tabular}

$I_{\max }$, total maximum plasma inhibitor concentration; NI, no inhibition.

${ }^{a}$ Risk assessment was based on the lowest IC $_{50}$ value, which was obtained with midazolam as a substrate.

substrate of OAT1 in vitro. In contrast, none of the tested concentrations of verinurad $(0.1-10 \mu \mathrm{M})$ showed more than $17 \%$ increase in cellular accumulation in MCDKII-OAT3 cells compared with control cells (Fig. 4B), indicating that verinurad is not a substrate of OAT3 in vitro.

In MDCKII-MATE1 and MDCKII-MATE2-K cells, uptake of verinurad was not significantly higher than in control cells at concentrations of $0.3-5 \mu \mathrm{M}(P>0.05)$ (Fig. $4, \mathrm{C}$ and D), indicating that verinurad is not a substrate of MATE1 and MATE2-K in vitro. Similarly, verinurad uptake was comparable in MDCKII-OCT2 cells and control cells at concentrations of $0.3-5 \mu \mathrm{M}$ (Fig. $4 \mathrm{E}$ ), indicating that verinurad is not a substrate of OCT2 in vitro.

Transport of Verinurad by Human Hepatic Uptake Transporters. Verinurad was found to undergo active hepatic uptake in suspended human hepatocytes, as there was a clear difference in uptake in the presence and absence of transporter inhibitors. It is worth noting that the inhibitors and concentrations used in the hepatocyte experiment primarily inhibit OCT1 and OATP transporters over other hepatic uptake transporters, including $\mathrm{Na}^{+}$/taurocholate cotransporting polypeptide and OAT2 (Bi et al., 2019). To elucidate the transporter(s) involved in the hepatic uptake, in vitro substrate studies were subsequently performed with OATP1B1, OATP1B3, OATP2B1, and OCT1 using MDCKII or CHO cells transfected with the respective transporter. Verinurad did not demonstrate OATP1B1-dependent uptake, with comparable uptake between MDCKII-OATP1B1-transfected cells and control cells at concentrations of $0.3-5 \mu \mathrm{M}$ or in the presence of rifampicin (Fig. 5A). In contrast, verinurad was identified as an OATP1B3 substrate in vitro, with statistically significant differences between MDCKIIOATP1B3 cells and control cells at $1 \mu \mathrm{M}(P=0.012)$ and 5 $\mu \mathrm{M}(P=0.0028)$ (Fig. 5B). The OATP1B3-mediated uptake of verinurad was inhibited by $100 \mu \mathrm{M}$ rifampicin, further confirming the role of OATP1B3 in the hepatic uptake (Fig. 5B).

In CHO-OATP2B1 cells, uptake of verinurad was comparable with that in control cells at concentrations of $0.3-5 \mu \mathrm{M}$ 
A

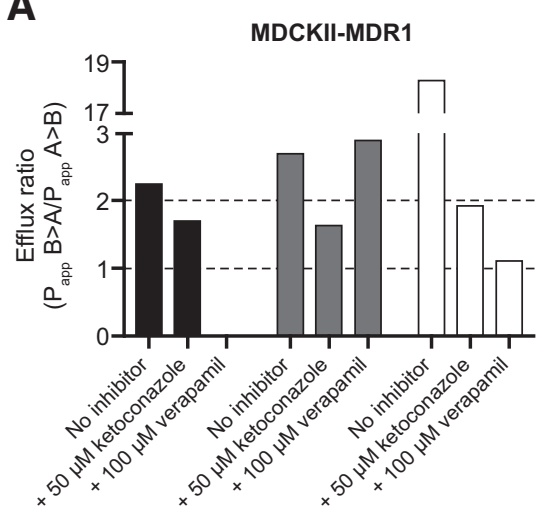

C

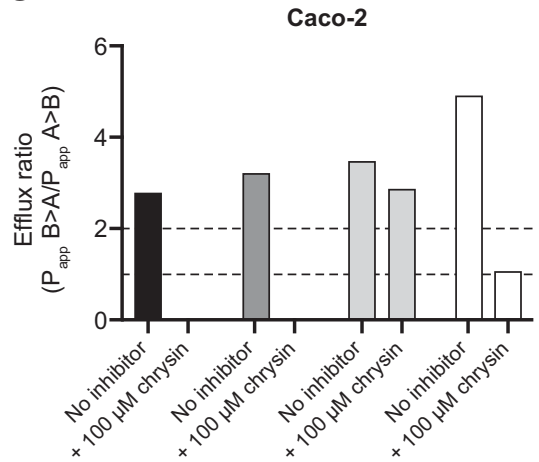

B
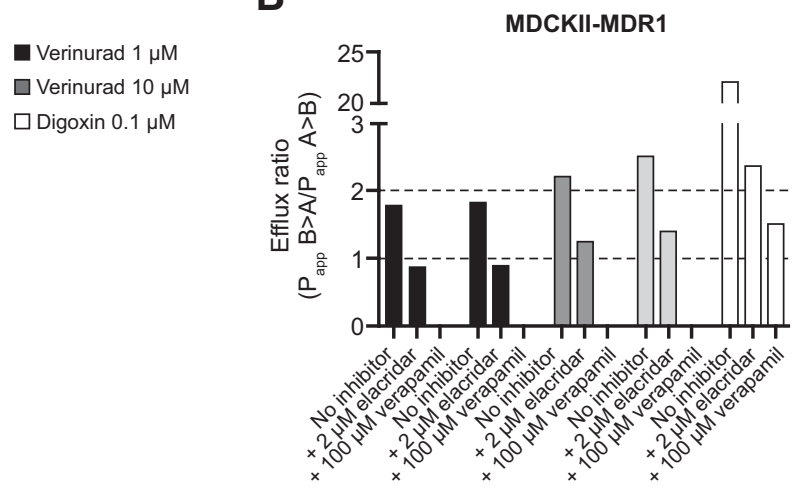

- Verinurad $1 \mu \mathrm{M}$

- Verinurad $3 \mu \mathrm{M}$

$\square$ Verinurad $10 \mu \mathrm{M}$

$\square$ Verinurad $30 \mu \mathrm{M}$

$\square$ Digoxin $0.1 \mu \mathrm{M}$

D
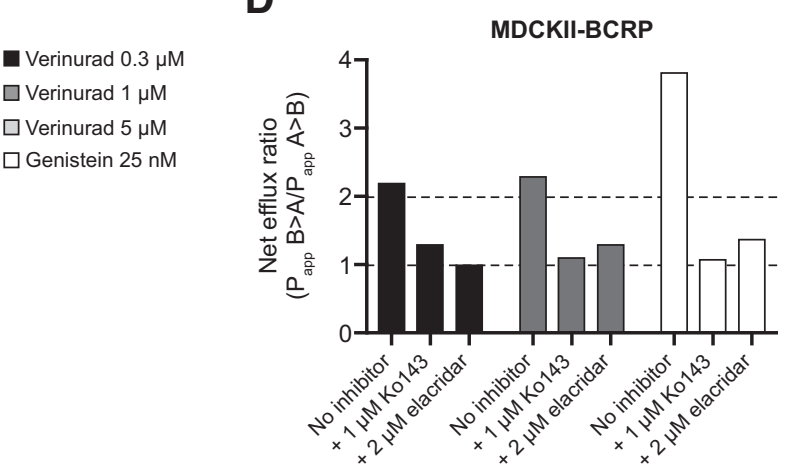

- Verinurad $0.5 \mu \mathrm{M}$ $\square$ Verinurad $2 \mu \mathrm{M}$ $\square$ Cimetidine $3 \mu \mathrm{M}$ $\square$ Genistein $25 \mathrm{nM}$

Fig. 2. Transcellular transport of verinurad by efflux transporters P-gp and BCRP. Efflux ratios of verinurad in the presence and absence of inhibitors in MDCKII-MDR1 cells (A and B), Caco-2 cells (C), and MDCKII-BCRP cells (D). Data are means of triplicate samples. $\mathrm{P}_{\text {app }}$, A > B, apparent permeability in apical to basolateral direction; $\mathrm{P}_{\text {app }} \mathrm{B}>\mathrm{A}$, apparent permeability in basolateral to apical direction.

(Fig. 5C), suggesting that verinurad is not a substrate of OATP2B1 in vitro. Similarly, verinurad uptake was comparable in MDCKII-OCT1 and control cells at concentrations of $0.3-5 \mu \mathrm{M}$ (Fig. 5D), indicating that verinurad is not a substrate of OCT1 in vitro.

In Vitro Evaluation of Verinurad, M1, and M8 as Inhibitors of Human Drug Transporters. The potential for verinurad and metabolites M1 and M8 to inhibit the transport of probe substrates by efflux (P-gp, BCRP, BSEP, MRP2, and MRP4), renal (OAT1, OAT3, MATE1, MATE2-K, and OCT2), and hepatic uptake transporters (OCT1, OAT2, OATP1B1, and OATP1B3) was determined in transporterexpressing cells or vesicles (Table 1, Supplemental Table 5). The resulting findings are summarized in Table 5 (efflux transporters), Table 6 (renal transporters), and Table 7 (hepatic uptake transporters).

In vitro, verinurad demonstrated concentration-dependent inhibition of OAT1, OATP1B1, OATP1B3, and BSEP activity, with $\mathrm{IC}_{50}$ values of $14.2,19.4,31.0$, and $109 \mu \mathrm{M}$, respectively (Tables 5-7). In addition, inhibition of OAT3-mediated transport was noted at the highest concentration tested $\left(\mathrm{IC}_{50}>100\right.$ $\mu \mathrm{M}$; Table 6). In contrast, verinurad did not inhibit the transport of probe substrates by P-gp, BCRP, OCT1, or OCT2 over the concentration range investigated $(0.3-100 \mu \mathrm{M}$; Tables $5-7)$.

Metabolites M1 and M8 inhibited MRP2-, OAT3-, and OATP1B1-dependent transport in vitro, with $\mathrm{IC}_{50}$ values of 1.13, 11.9, and 3.07 $\mu \mathrm{M}$ for $\mathrm{M} 1$ and 5.50, 30.4, and $19.8 \mu \mathrm{M}$ for M8, respectively (Tables 5-7). M1 also inhibited BCRP and OATP1B3 with $\mathrm{IC}_{50}$ values of 145 and $18.4 \mu \mathrm{M}$, respectively, whereas inhibition of these transporters by M8 was insufficient to accurately determine $\mathrm{IC}_{50}$ values $\left(\mathrm{IC}_{50}>100 \mu \mathrm{M}\right.$; Table 5 and 7). Less than $50 \%$ inhibition of OAT1 activity by M1 and M8 and of MATE1 and MATE2-K activity by M1 was also observed $\left(\mathrm{IC}_{50}>100 \mu \mathrm{M}\right)$, whereas M8 did not inhibit the transport of probe substrates by either MATE1 or MATE2-K (Table 6). Likewise, M1 inhibited MRP4 at the highest concentration tested $\left(\mathrm{IC}_{50}>10 \mu \mathrm{M}\right)$, whereas M8 did not demonstrate concentration-dependent inhibition of MRP4 over the concentration range investigated (0.1-10 $\mu \mathrm{M}$; Table 5). Similar to verinurad, neither M1 nor M8 inhibited the active transport by P-gp, OCT1, and OCT2 (Tables 5-7).

\section{Discussion}

Verinurad is in development for CKD and HFpEF, which are associated with comorbidities and frequent polypharmacy. Assessment of the potential for clinically relevant DDIs with verinurad early in development is therefore essential. The present studies characterized the in vitro DDI potential of verinurad and metabolites M1 and M8 as victims and perpetrators of key metabolizing enzymes and drug transporters. Our results provide new insights on verinurad's disposition in humans (Fig. 6) and inform phase 2 and 3 protocols and inclusion and exclusion criteria. 
A

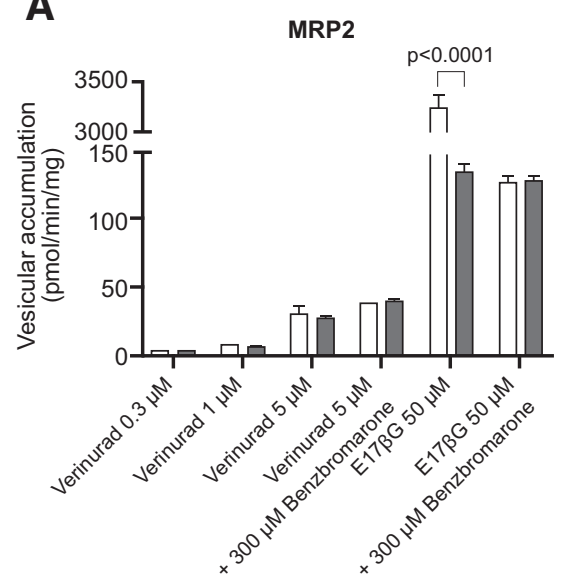

C

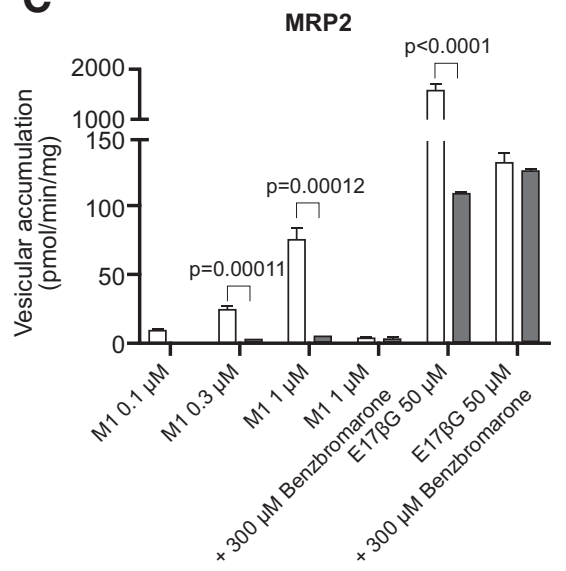

E

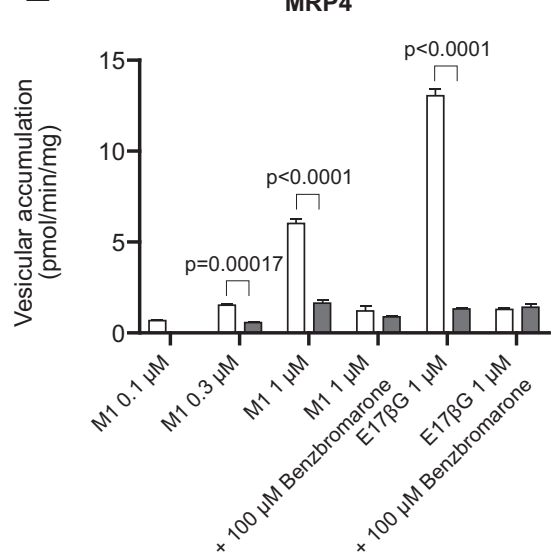

B
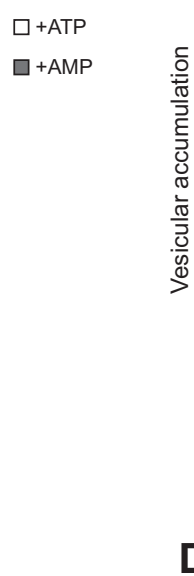

$\square+$ ATP

$\square+$ AMP

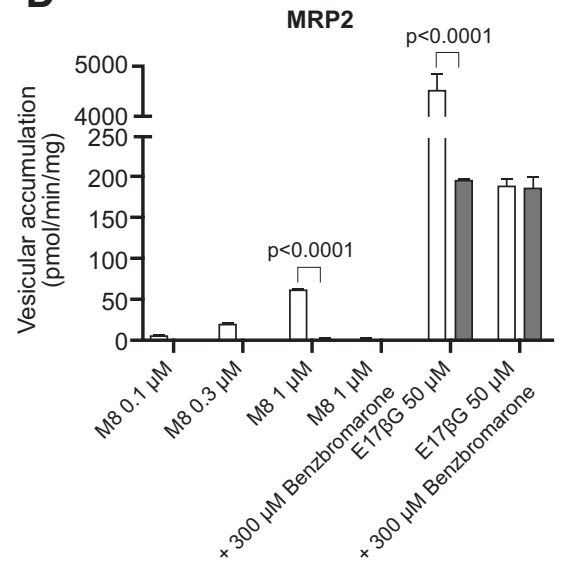

F
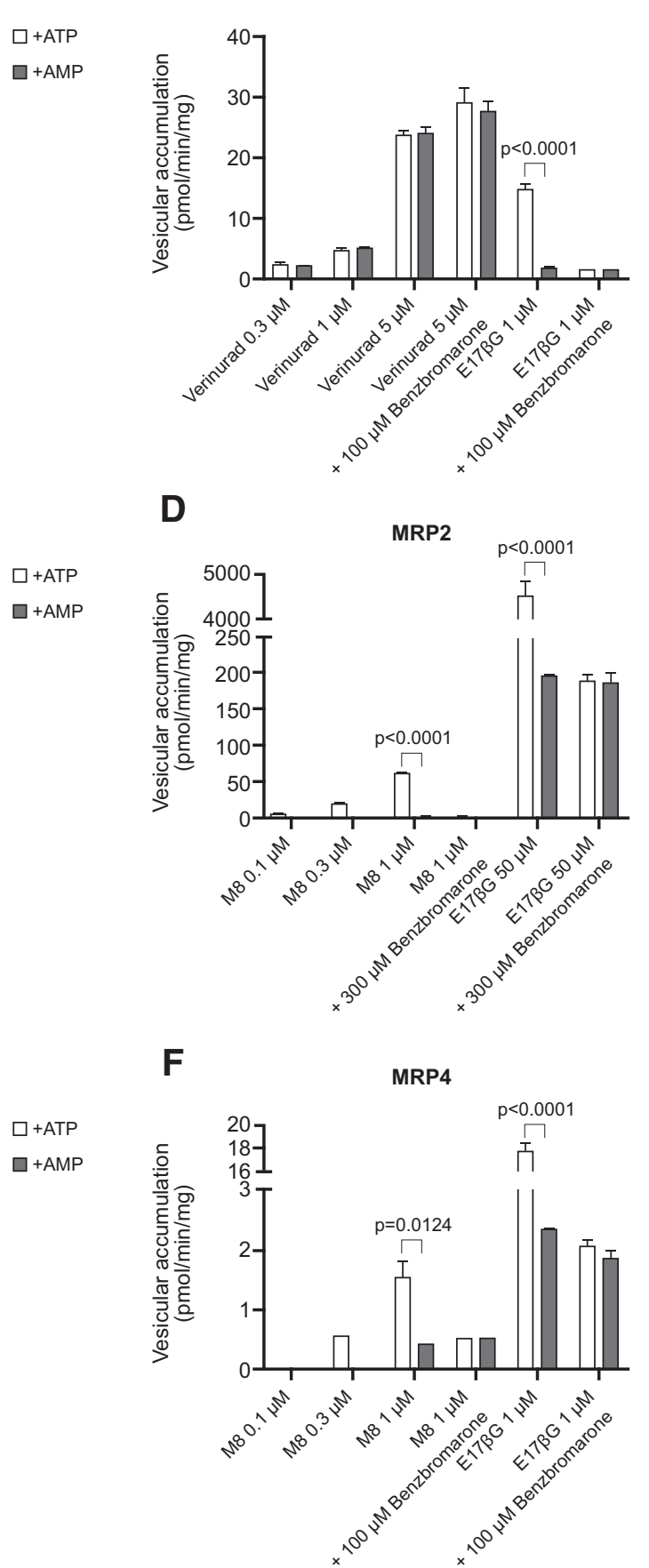

$\square+$ ATP

$\square+$ AMP

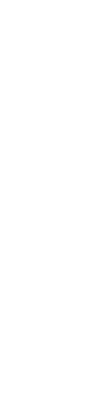

$\square+$ ATP

$\square+$ AMP

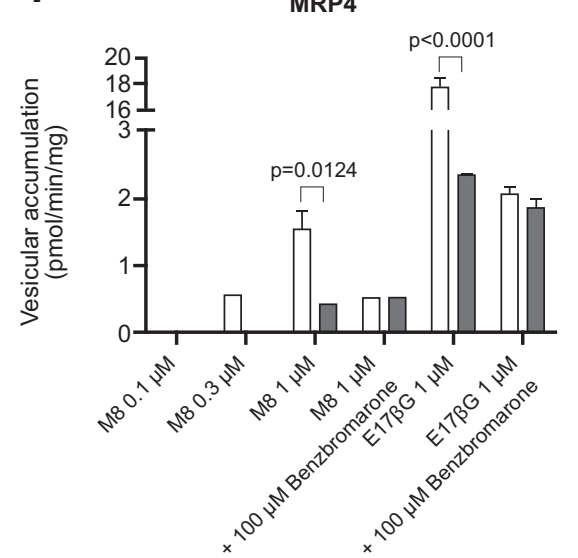

Fig. 3. Transport of verinurad and metabolites M1 and M8 by efflux transporters MRP2 and MRP4. Uptake of verinurad stimulated by ATP or AMP in Sf9 vesicles expressing MRP2 (A) and MRP4 (B). Uptake of M1 (C) and M8 (D) stimulated by ATP or AMP in Sf9 vesicles expressing MRP2. Uptake of M1 (E) and M8 (F) stimulated by ATP or AMP in Sf9 vesicles expressing MRP4. Data are mean and standard deviation of triplicate samples. Unpaired Student's $t$ test was used to assess the difference in ATP- and AMP-stimulated uptake. A p-value of $<0.05$ was considered statistically significant. ATP, adenosine triphosphate; AMP, adenosine monophosphate; Sf9, Spodoptera frugiperda subclone 9.

The inhibitory potencies of verinurad and metabolites M1 and M8 on major CYP enzymes were examined in vitro using drug substrates recommended by regulatory agencies and reported by the scientific community. For verinurad, $\mathrm{IC}_{50}$ values could only be obtained for CYP2C8, CYP2C9, and CYP3A4/5. However, the calculated ratios of intrinsic clearance values $\left(R_{1}\right)$ were $<1.02$ for systemic interactions with
CYP2C8, CYP2C9, and CYP3A4/5, and the calculated $\mathrm{R}_{1 \text {, out }}$ for CYP3A4/5 (4.6) was below the risk threshold of 11 for intestinal CYP3A4/5 interactions, indicating a low risk of clinically relevant DDIs with verinurad as a direct inhibitor of CYP2C8, CYP2C9, and CYP3A4/5. Neither M1 nor M8 produced direct inhibition of any metabolizing enzyme tested, and there was no evidence of TDI of CYP1A2, CYP2C8, CYP2C9, 
A

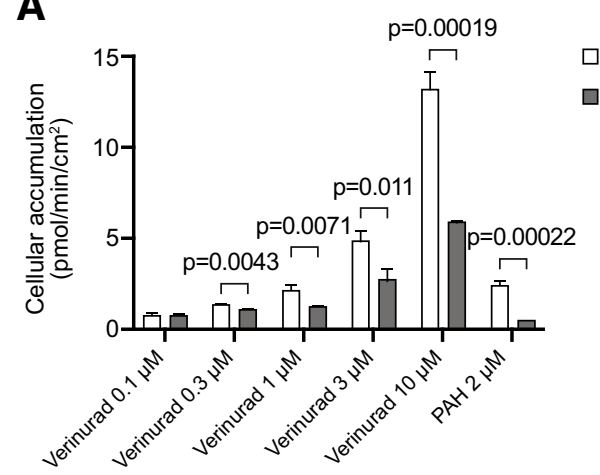

C

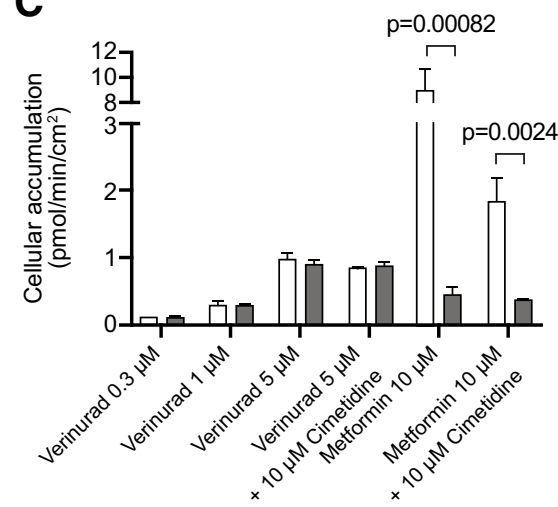

E

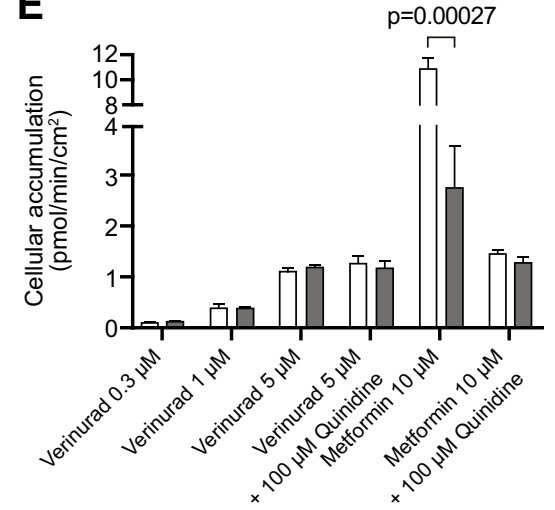

B

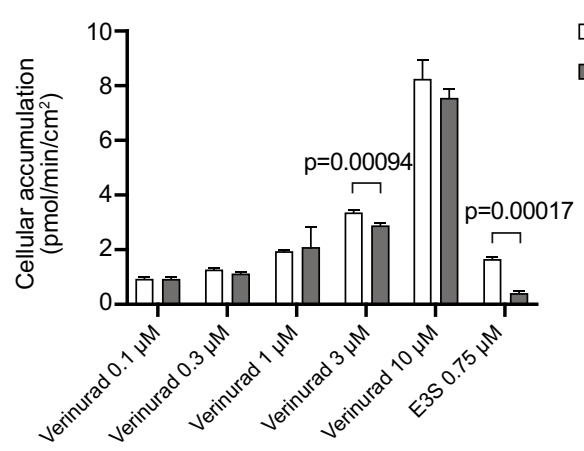

D

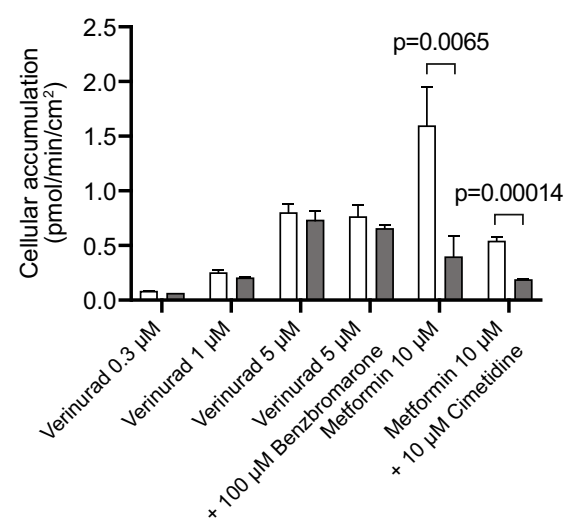

$\square$ MDCKII-OAT3

$\square$ MDCKII-contro

MDCKII-control

$\square$ MDCKII-MATE1

$\square$ MDCKII-control

$\square$ MDCKII-OCT2

$\square$ MDCKII-control

Fig. 4. Transport of verinurad by human renal transporters. Uptake of verinurad in MDCKII-OAT1 (A), MDCKII-OAT3 (B), MDCKII-MATE1 (C), MDCKII-MATE2-K (D), and MDCKII-OCT2 (E) cells as compared with uptake in control cells. Data are mean and S.D. of triplicate samples. Unpaired Student's $t$ test was used to assess the difference in uptake in transporter-expressing and control cells. A p-value of $<0.05$ was considered statistically significant.

CYP2C19, CYP2D6, or CYP3A4/5 with verinurad, M1, or M8. This was an interesting finding with respect to CYP2C8 and one that is in contrast with studies showing that some acyl glucuronides, such as those of clopidogrel, gemfibrozil, and deleobuvir, are direct and time-dependent inhibitors of CYP2C8 (Shitara et al., 2004; Ogilvie et al., 2006; Tornio et al., 2014; Backman et al., 2016; Kim et al., 2016; Sane et al., 2016; Ma et al., 2017). Although not recommended explicitly by regulatory agencies, we also assessed the systemic (hepatic) and intestinal UGT interactions for verinurad as described for CYP-mediated interactions and observed a low risk of clinically relevant DDIs with verinurad as a direct inhibitor of UGT1A1 and UGT2B7. Overall, there is a low potential for verinurad and metabolites M1 and M8 to inhibit CYP and UGT enzymes at clinically relevant concentrations.
In primary human hepatocytes, verinurad did not induce activity or mRNA expression of CYP1A2, CYP2B6, or CYP3A4/5 compared with prototypical inducers and was below the 20\% threshold from the US FDA (Food and Drug Administration, 2020). Consistent with the absence of reports in the literature to implicate acyl glucuronides as CYP inducers, it is likely that M1 and M8 are not inducers of CYP1A2, CYP2B6, or CYP3A4/5 either.

Our collective studies showed that verinurad was a substrate of the efflux transporters P-gp and BCRP in vitro. However, the finding that efflux of verinurad was not reduced by verapamil in a similar manner as with ketoconazole in the MDCKII-MDR1 cell line means that we cannot exclude the potential contribution of canine P-gp to the lack of P-gp inhibitory effect of verapamil. Conceptually, P-gp and BCRP may 
A

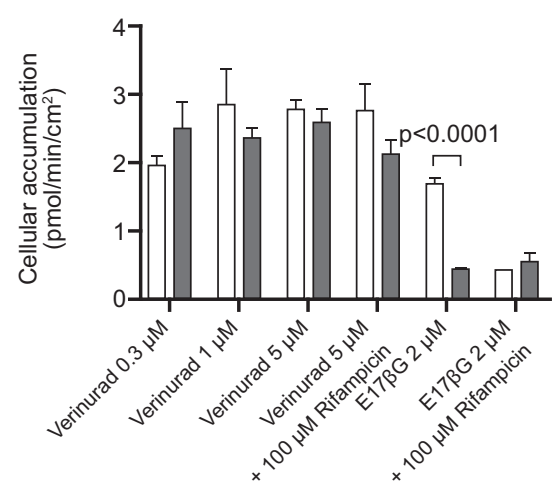

C

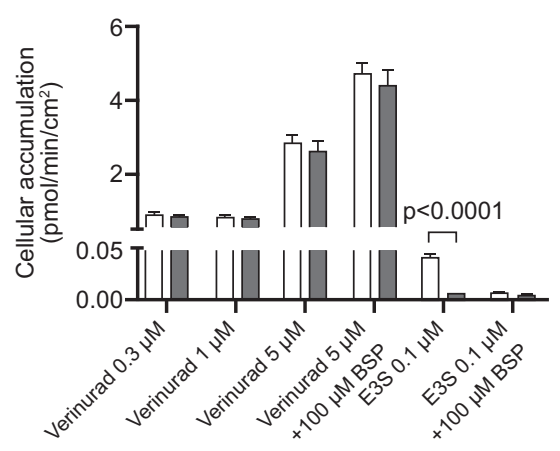

B
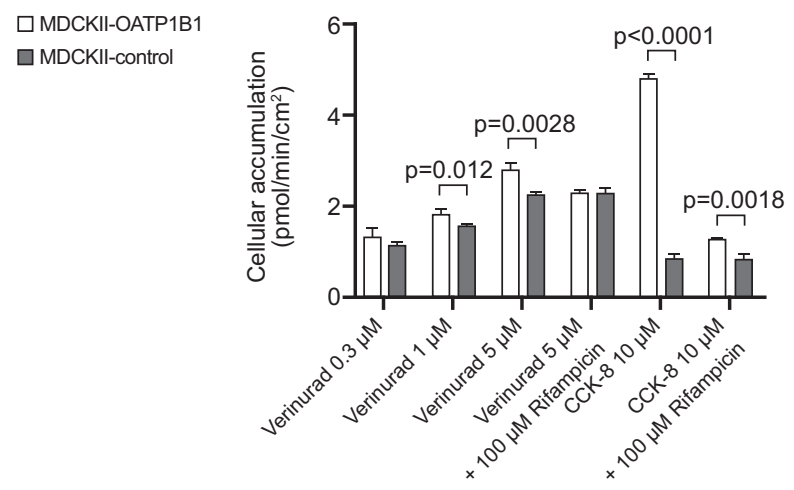

D

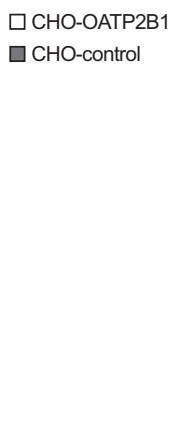

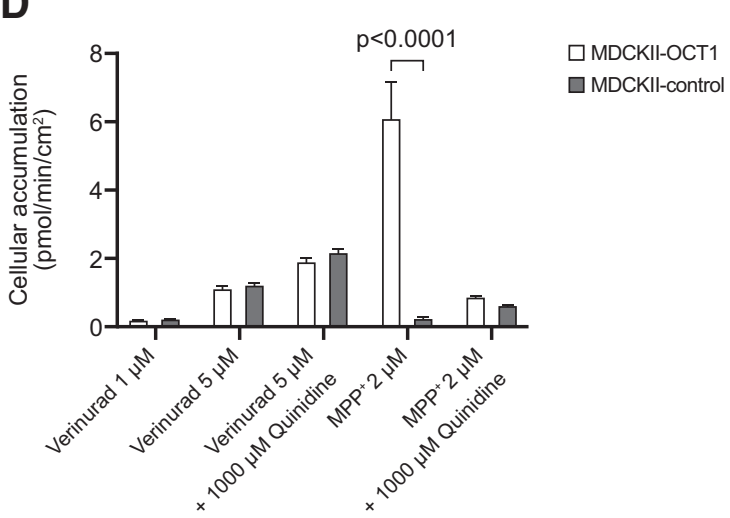

Fig. 5. Transport of verinurad by human hepatic uptake transporters. Cellular accumulation of verinurad in MDCKII-OATP1B1 (A), MDCKIIOATP1B3 (B), CHO-OATP2B1 (C), and MDCKII-OCT1 (D) cells as compared with control cells. Data are mean and S.D. of triplicate samples. Unpaired Student's $t$ test was used to assess the difference in uptake in transporter-expressing and control cells. A p-value of $<0.05$ was considered statistically significant. BSP, bromosulfophthalein; CHO, Chinese hamster ovary.

impact the oral bioavailability, tissue distribution, and hepatic and renal elimination of their substrates (Zhang et al., 2018). However, because of its high solubility and high intrinsic permeability, verinurad absorption is not impacted by intestinal efflux transporters, as reflected in its fraction absorbed of $\geq 64 \%$ (Lee et al., 2018) and dose-proportional pharmacokinetics up to $40 \mathrm{mg}$ after a single dose (Shen et al., 2017) and multiple once-daily doses (Hall et al., 2018). Collectively, this suggests that P-gp and BCRP are likely to have minimal impact on the risk of clinically relevant DDIs.

Hepatic disposition of verinurad involves a combination of active uptake and passive diffusion followed by metabolism and efflux (Fig. 6). Our in vitro data revealed that verinurad is a substrate of OATP1B3 but not of other OATP transporters unlike typical anionic compounds or carboxylic acid moieties, which are common substrates shared by multiple OATP-isoforms (Kalgutkar and Daniels, 2010). In vitro, verinurad uptake into hepatocytes showed almost equal active transport and passive diffusion at the lower concentration and largely active uptake at the higher concentration. This unexpected finding made it challenging to deduce the clinical role of active hepatic uptake in the disposition of verinurad. Therefore, the OATP DDI potential will be further elucidated in a clinical DDI study (study D5495C00013 [NCT04532918]).

Verinurad was also identified as a substrate of OAT1 in vitro. Metabolites M1 and M8 are the main components of the verinurad dose eliminated in urine, whereas $<2 \%$ of verinurad is excreted unchanged renally (Lee et al., 2018), and the contribution is expected to be even lower in renally impaired patients. Therefore, OAT1 inhibition is not expected to significantly alter the pharmacokinetics of verinurad. In renal proximal tubular cells, CYP and UGTs can contribute to the formation of M1 and M8, which can then be effluxed by MRP2/4 into the urine (Fig. 6). Although urinary excretion decreases with reduced renal function, evidence from a singledose renal impairment study supports the safety and tolerability of higher concentrations of M1 and M8 observed in severe renal impairment (Smith et al., 2018). Therefore, the risk of comedications that may alter uptake by OAT1 and change renal clearance of verinurad itself is low, but inhibitors or inducers of MRP2 may impact the pharmacokinetics of verinurad by altering the elimination of metabolites (Marschall et al., 2005; Oscarson et al., 2007).

In our in vitro transporter inhibition studies, verinurad did not inhibit P-gp, BCRP, OCT1, OCT2, or OAT3, whereas M1 and M8 did not inhibit P-gp, MRP4, OCT1, MATE1, MATE2K, or OCT2. Applying established static equations with the moderate inhibitory potencies observed for OATPs or OAT1 together with the estimated worst case $\mathrm{C}_{\max }$ of verinurad and metabolites based on the highest dose tested in phase 2 clinical studies, the overall risk for clinically relevant pharmacokinetic DDIs via inhibition of the efflux, renal, and hepatic transporters studied is considered low, except for the previously described pharmacological effect on oxypurinol 
TABLE 5

In vitro evaluation of inhibition of efflux transporters by verinurad and metabolites M1 and M8

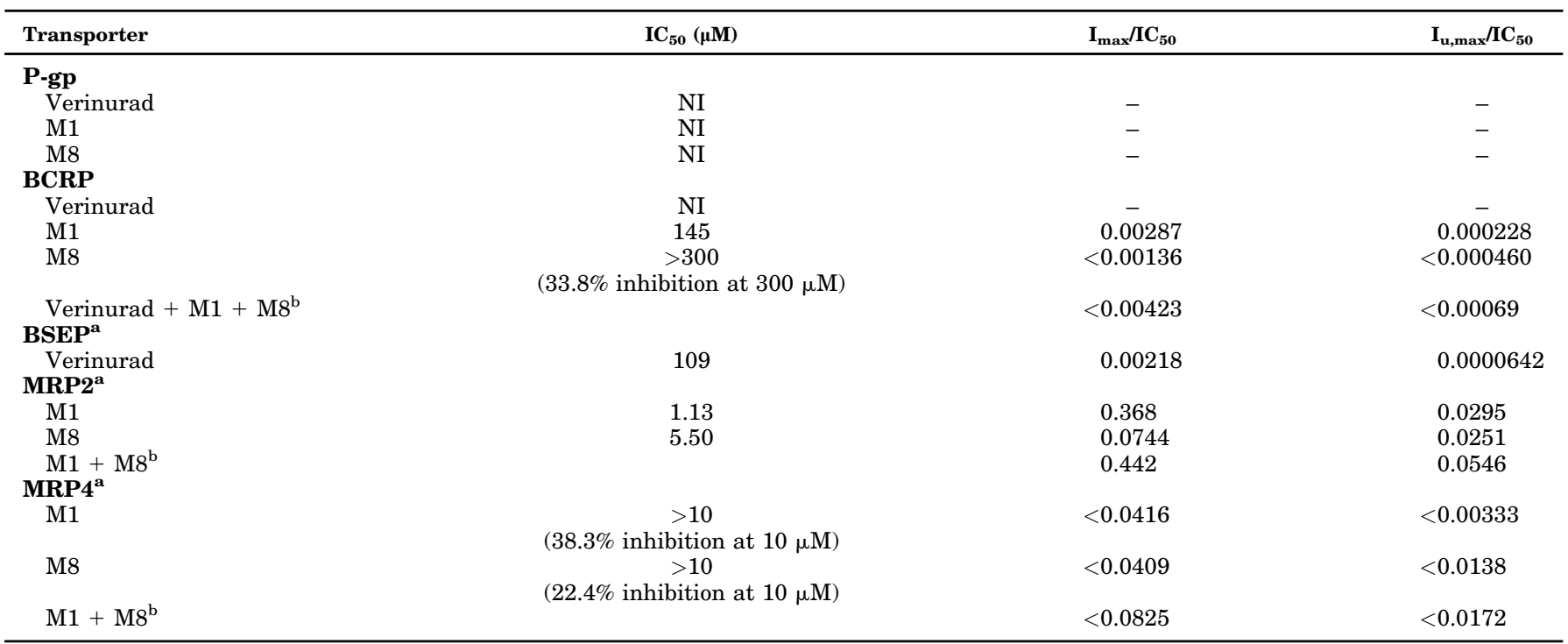

$\mathrm{C}_{\max }$, maximum observed concentration; EMA, European Medicines Agency; FDA, Food and Drug Administration; $\mathrm{I}_{\text {max }}$, total maximum plasma inhibitor concentration; $I_{\mathrm{u}, \max }$, maximum free plasma inhibitor concentration; NI, no inhibition.

${ }^{a}$ Estimated margin in analogy to efflux transporter inhibition concept in US FDA 2020 and EMA guidance, 10 -fold total $\mathrm{C}_{\text {max }}$ (US FDA): $\mathrm{R}=\mathrm{I}_{\text {max }} / \mathrm{IC}_{50} \geq 0.1 ; 50$-fold unbound $\mathrm{C}_{\max }$ (EMA): $\mathrm{I}_{\mathrm{u}, \max } / \mathrm{IC}_{50} \geq 0.02$.

${ }^{\mathrm{b}}$ The combined inhibitory potential of verinurad and its metabolites was calculated as a sum of the individual ratios of exposure in relation to in vitro inhibition constants.

elimination through inhibition of URAT1, which is itself a renal transporter (Kankam et al., 2018).

A unique aspect of our studies is the assessment of in vitro DDI potentials of metabolites M1 and M8 as victims and perpetrators of drug transporters. Acyl glucuronides M1 and M8 were substrates of MRP2 and MRP4 in vitro. Efflux via MRPs contributes to the disposition of several glucuronide conjugates, for example, those of mycophenolic acid (Westley et al., 2006; Patel et al., 2013), atorvastatin (Rodrigues et al., 2020), and diclofenac (Zhang et al., 2016), in which MRP4 contributes

TABLE 6

In vitro evaluation of inhibition of human renal transporters by verinurad and metabolites M1 and M8

\begin{tabular}{|c|c|c|}
\hline Transporter & $\mathrm{IC}_{50}(\mu \mathrm{M})$ & $\mathbf{I}_{\mathbf{u}, \max } / \mathbf{K}_{\mathbf{i}}^{\mathbf{a}}$ \\
\hline \multicolumn{3}{|l|}{ OCT2 } \\
\hline Verinurad & NI & _- \\
\hline M1 & NI & - \\
\hline \multicolumn{3}{|l|}{ OAT1 } \\
\hline Verinurad & 14.2 & 0.00048 \\
\hline M1 & $>100$ & $<0.00033^{\mathrm{b}}$ \\
\hline & (47.1\% inhibition at $100 \mu \mathrm{M})$ & \\
\hline Verinurad $+\mathrm{M} 1+\mathrm{M} 8^{\mathrm{c}}$ & & $<0.00219$ \\
\hline \multicolumn{3}{|l|}{ OAT3 } \\
\hline Verinurad & $\begin{array}{c}>100 \\
(19.3 \% \text { inhibition at } 100 \mu \mathrm{M})\end{array}$ & $<0.000068^{\mathrm{b}}$ \\
\hline M1 & 11.9 & 0.00559 \\
\hline M8 & 30.4 & 0.00914 \\
\hline Verinurad $+\mathrm{M} 1+\mathrm{M} 8^{\mathrm{c}}$ & & $<0.0148$ \\
\hline \multicolumn{3}{|l|}{ MATE1 } \\
\hline M1 & $>100$ & $<0.00033^{\mathrm{a}}$ \\
\hline & $(48.8 \% \text { inhibition at } 100 \mu \mathrm{M})^{\mathrm{a}}$ & \\
\hline M8 & NI & - \\
\hline
\end{tabular}

$I_{u, \max }$, maximum free plasma concentration; $K_{i}$, inhibitory constant; $K_{m}$, Michaelis-Menten constant; NI, no inhibition.

${ }^{a}$ In inhibition studies, probe substrate concentration was sufficiently lower than its $\mathrm{K}_{\mathrm{m}}$ such that $\mathrm{IC}_{50}$ approximates to $\mathrm{K}_{\mathrm{i}}$

${ }^{\mathrm{b}}$ Risk margin estimate based on highest drug concentration in the assay (which yielded $<50 \%$ inhibition).

${ }^{\mathrm{c}}$ The combined inhibitory potential of verinurad and its metabolites was calculated as a sum of the individual ratios of exposure in relation to in vitro inhibition constants. 
TABLE 7

In vitro evaluation of inhibition of human hepatic uptake transporters by verinurad and metabolites M1 and M8

\begin{tabular}{|c|c|c|c|c|}
\hline Transporter & $\mathrm{IC}_{50}(\mu \mathrm{M})$ & $\mathbf{I}_{\mathrm{in}, \max }(\mu \mathbf{M})^{\mathbf{a}}$ & $\mathbf{I}_{\mathbf{u}, \mathbf{i n}, \max } / \mathbf{K}_{\mathbf{i}}^{\mathbf{b}}$ & $\mathbf{R}^{\mathbf{c}}$ \\
\hline \multicolumn{5}{|l|}{ OATP1B1 } \\
\hline Verinurad & 19.4 & 7.89 & 0.0233 & 1.023 \\
\hline M1 & 3.07 & 0.694 & 0.0322 & 1.032 \\
\hline M8 & 19.8 & 0.682 & 0.0207 & 1.021 \\
\hline Verinurad $+\mathrm{M} 1+\mathrm{M} 8^{\mathrm{d}}$ & & & 0.0762 & 1.076 \\
\hline \multicolumn{5}{|l|}{ OATP1B3 } \\
\hline Verinurad & 31.0 & 7.89 & 0.00365 & 1.004 \\
\hline M1 & 18.4 & 0.694 & 0.00302 & 1.003 \\
\hline M8 & $\begin{array}{c}>100 \\
(40.6 \% \text { inhibition at } \\
100 \mu \mathrm{M})\end{array}$ & 0.682 & $<0.00230$ & $<1.002$ \\
\hline \multirow{2}{*}{\multicolumn{5}{|c|}{ OAT2 }} \\
\hline & & & & \\
\hline Verinurad & $\begin{array}{c}>100 \\
(33.1 \% \text { inhibition at } 100 \mu \mathrm{M})\end{array}$ & 7.89 & $<0.00453$ & $<1.005$ \\
\hline \multicolumn{5}{|c|}{ (J3.1\% innioition at $100 \mu \mathrm{Mv})$} \\
\hline Verinurad & NI & - & - & - \\
\hline M1 & NI & - & - & - \\
\hline M8 & NI & - & - & - \\
\hline
\end{tabular}

$\mathrm{C}_{\max }$, maximum observed concentration; FDA, Food and Drug Administration; $\mathrm{f}_{\mathrm{u}}$, unbound fraction in plasma; $\mathrm{I}_{\mathrm{in}, \max }$, maximum hepatic inlet concentration; $\mathrm{I}_{\max }$, total maximum plasma inhibitor concentration; $I_{u, \text { in,max }}$, maximum free hepatic inlet concentration; $K_{i}$, inhibitory constant; $N C$, not calculated.

${ }^{\mathrm{a}} \mathrm{I}_{\mathrm{in}, \max }$ calculated based on US FDA 2020 guidance. $\mathrm{I}_{\mathrm{in}, \max }=\mathrm{I}_{\max }+\left(\mathrm{F}_{\mathrm{a}} \times \mathrm{F}_{\mathrm{g}} \times \mathrm{K}_{\mathrm{a}} \times \operatorname{dose} /\left(\mathrm{Q}_{\mathrm{h}}\right) /(\mathrm{B}: \mathrm{P})=0.238 \mu \mathrm{M}+[(1 \times 1 \times 0.1 \times 68.9 \mathrm{mmol} / 1.5 / 0.6=7.654]=\right.$ 7.89 , in which dose $=24 \mathrm{mg}=68.9 \mu \mathrm{mol}, \mathrm{Q}_{\mathrm{h}}=1.5 \mathrm{l} / \mathrm{min}$, and $\mathrm{B}: P=0.6$. For metabolites, $\mathrm{I}_{\mathrm{in}, \max }$ is estimated from total $\mathrm{C}_{\max }$ and $\mathrm{Rb}$ of $0.6-0.693 \mu \mathrm{M}$ and 0.682 $\mu \mathrm{M}$ for $\mathrm{M} 1$ and $\mathrm{M} 8$, respectively.

${ }^{\mathrm{b}} \mathrm{K}_{\mathrm{i}}$ was estimated to equal $\mathrm{IC}_{50}$ when substrate concentration was well below the reported $\mathrm{K}_{\mathrm{m}}$ or as $\mathrm{IC}_{50} / 2$ when substrate concentration was close to $\mathrm{K}_{\mathrm{m}}$.

${ }^{\mathrm{c}} \mathrm{R}=1+\left(\left(\mathrm{f}_{\mathrm{u}} \times \mathrm{I}_{\mathrm{in}, \max }\right) / \mathrm{IC}_{50}\right) \geq 1.1$

$\mathrm{d}$ The combined inhibitory potential of verinurad and its metabolites was calculated as a sum of the individual ratios of exposure in relation to in vitro inhibition constants.

to basolateral hepatic efflux and MRP2 contributes to biliary excretion that can lead to enterohepatic recirculation after hydrolysis by $\beta$-glucuronidases and intestinal reabsorption.
This reuptake route may explain the small secondary peak observed in the plasma exposure profile after verinurad dosing (Lee et al., 2018). Transporter inhibition by cyclosporine

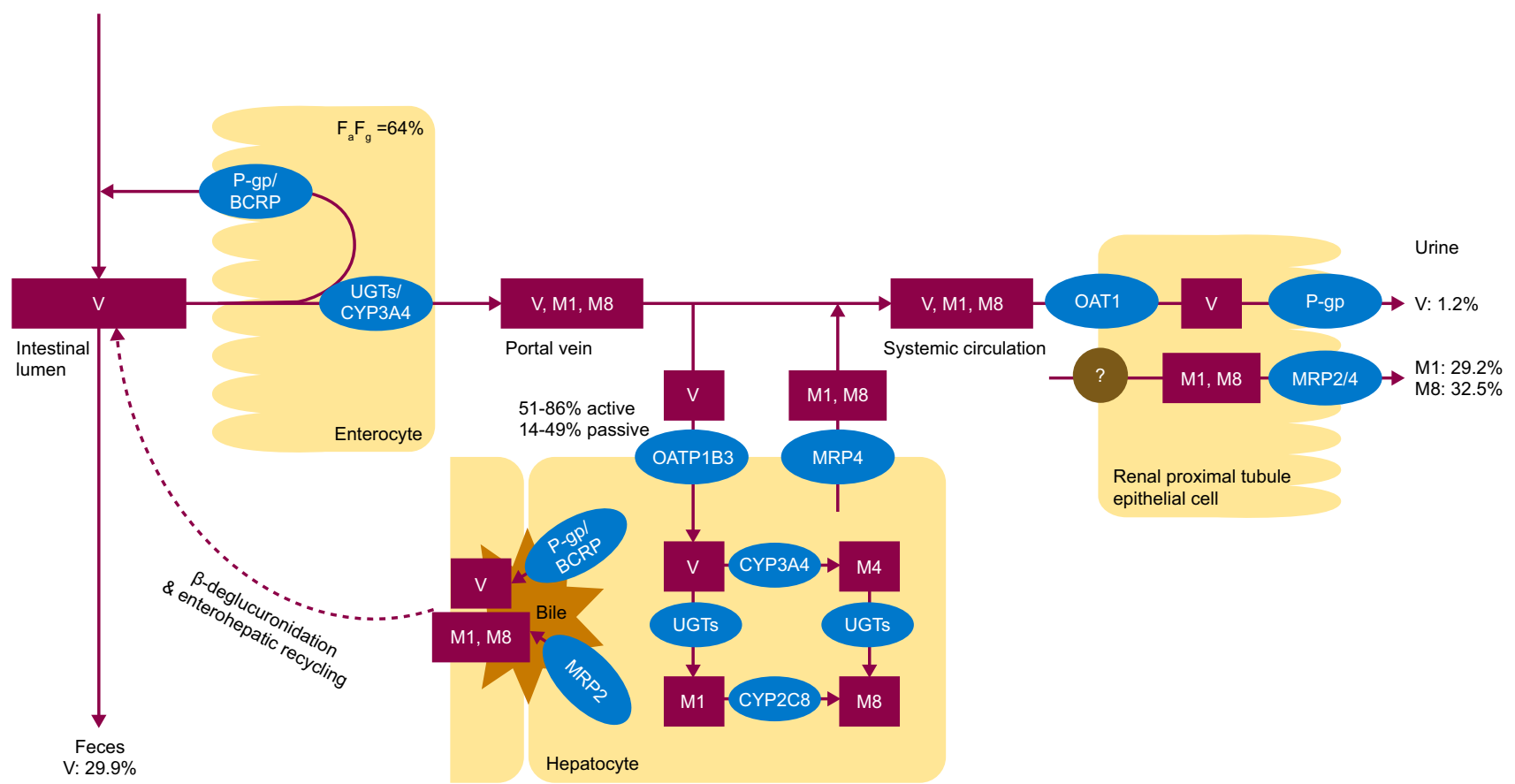

Fig. 6. The hypothesized mechanisms of disposition of verinurad and metabolites M1 and M8. After oral administration of verinurad, metabolism to form M4, M1, and M8 may occur in enterocytes, as CYP3A4/UGT enzymes are present. Verinurad and metabolite(s) enter the portal vein, and verinurad is taken up by the hepatocytes in the liver via passive diffusion and active transport by OATP1B3. In the hepatocytes, verinurad is metabolized two ways: 1) to M4 (by CYP3A4) and then subsequently conjugated by UGTs to form M8 and 2) to M1 (by UGTs), which may be sequentially metabolized to M8 by CYP2C8. In addition, P-gp and BCRP may contribute to hepatic efflux of verinurad to the bile. Metabolites that undergo enterohepatic recycling are effluxed to the bile via MRP2 and undergo complete deconjugation by $\beta$-glucuronidase to release the aglycone to enter the portal system to the liver. Metabolites effluxed by MRP4 to the systemic circulation can be eliminated by the kidney and excreted in the urine or can be taken up by the liver and undergo enterohepatic recycling. Verinurad is also eliminated by the kidney to a limited extent, possibly via OAT1-mediated uptake and P-gp-mediated efflux across the renal proximal tubular epithelial cells. $\mathrm{F}_{\mathrm{a}} \mathrm{F}_{\mathrm{g}}$, fraction of the dose of inhibitor that is absorbed; M1, acyl glucuronide of verinurad; M4, $N$-oxide of verinurad; M8, acyl glucuronide of verinurad- $N$-oxide; UGT, glucuronosyl transferases; V, verinurad. 
decreases the AUC of mycophenolic acid by disrupting the enterohepatic recycling process (Westley et al., 2006). By the same analogy, the pharmacokinetics of verinurad may be altered by comedications that inhibit MRPs.

In our in vitro transporter inhibition studies, only MRP2 was inhibited by $\mathrm{M} 1$ and $\mathrm{M} 8$ in vitro with $\mathrm{IC}_{50}$ values 3 - to 13 fold above projected therapeutic $\mathrm{C}_{\max }$, respectively. Guidance from regulatory agencies do not provide a framework for assessing the risk for clinically relevant MRP2 inhibition; however, the potential for DDIs can be explored by applying the assessment approaches for other efflux transporters. For instance, both M1 and M8 meet the threshold for potential DDIs described by the US FDA for MATE based on a 10-fold margin for the free plasma concentration (Food and Drug Administration, 2020). Yet, both M1 and M8 were above (34and 40-fold margin, respectively) the threshold for potential DDIs described by the EMA for P-gp based on a 50-fold threshold for free plasma concentration (European Medicines Agency, 2013). Additionally, the US FDA and JPMDA use a 10-fold margin for P-gp, and our in vitro data indicate that the total $\mathrm{I}_{\text {max }} \mathrm{IC}_{50}$ for $\mathrm{M} 1$ is lower than this threshold, but for M8 it is higher (Japanese Pharmaceuticals and Medical Devices Agency, 2018; Food and Drug Administration, 2020). Therefore, metabolites M1 and M8 cannot be fully excluded as inhibitors of MRP2 based on risk assessment approaches for other efflux transporters. MRP2 substrates are often conjugated drug metabolites (Hillgren et al., 2013) whose clearance may be modulated by inhibition of MRP2 (Westley et al., 2006; Patel et al., 2013; Picard, 2013) but compensated by efflux through other MRP isoforms, such as MRP3 or MRP4 (Hillgren et al., 2013; Zhang et al., 2016). Overall, the clinical interpretation of our findings is complicated by an absence of a specific substrate of MRP2 to evaluate inhibition in vivo. Furthermore, there is neither evidence in the literature for clinically significant inhibition of MRP2 that includes MRP2 polymorphisms or specific pharmacokinetic-related substrates (Suzuki and Sugiyama, 2002; Niemi et al., 2006; Hillgren et al., 2013) nor specific guidance provided by regulatory agencies.

Besides the individual DDI risk assessments for verinurad, M1, and M8, we also assessed the combined inhibitory potential of verinurad and its metabolites by calculating additive $\mathrm{R}$ values (see Materials and Methods section for details). Except for OATP1B1, in which the FDA threshold for potential DDI risk was met, whereas the EMA threshold was not, our calculations indicated no further risk when characterizing the combined inhibitory effect of verinurad and its metabolites.

In conclusion, the systematic quantitative risk analysis of in vitro data conducted here indicates that apart from verinurad inhibiting URAT1 and being a substrate of OATP1B3, the potential for clinically relevant DDIs for verinurad and metabolites M1 and M8 as victims or perpetrators of metabolizing enzymes or drug transporters is low. A clinical study is ongoing to assess the relevance of OATP engagement in the pharmacokinetics of verinurad. Following recent US FDA guidance, a unique aspect of this report is the evaluation of the nonactive acyl glucuronide metabolites, which were identified as substrates and inhibitors of MRPs.

\section{Acknowledgments}

The authors thank Chad Elmore of AstraZeneca for ensuring the purity of synthetic materials. Medical writing support was provided by Shaun W. Foley, BSc (Hons) CMPP, and editorial support was provided by Bethany King, BSc (Hons), all of Core Medica, London, UK, supported by AstraZeneca according to Good Publication Practice guidelines. The sponsor was involved in the study design, collection, analysis, and interpretation of data as well as data checking of information provided in the manuscript. Ultimate responsibility for opinions, conclusions, and data interpretation lies with the authors.

\section{Authorship Contributions}

Participated in research design: Gopaul, Lee.

Conducted experiments: Lee.

Performed data analysis: Gopaul, Vildhede, Andersson, Erlandsson, Lee, Johansson, Hilgendorf.

Wrote or contributed to the writing of the manuscript: Gopaul, Vildhede, Andersson, Erlandsson, Lee, Johansson, Hilgendorf.

\section{References}

Backman JT, Filppula AM, Niemi M, and Neuvonen PJ (2016) Role of cytochrome P450 2C8 in drug metabolism and interactions. Pharmacol Rev 68:168-241.

Bi Y-a, Costales C, Mathialagan S, West M, Eatemadpour S, Lazzaro S, Tylaska L, Scialis R, Zhang H, Umland J, Kimoto E, Tess DA, Feng B, Tremaine L, Varma MVS, and Rodrigues AD (2019) Quantitative contribution of six major transporters to the hepatic uptake of drugs: 'SLC-phenotyping' using primary human hepatocytes. Journal of Pharmacology and Experimental Therapeutics:jpet.119.257600.

Carnicelli AP, Clare R, Chiswell K, Lytle B, Bjursell M, Perl S, Andersson K, Hedman K, Pagidipati N, Vemulapalli S, et al. (2020a) Comparison of characteristics and outcomes of patients with heart failure with preserved ejection fraction with versus without hyperuricemia or gout. Am J Cardiol 127:64-72.

Carnicelli AP, Sun J-L, Alhanti B, Bjursell M, Perl S, Lytle B, Roe MT, and Mentz RJ (2020b) Elevated uric acid prevalence and clinical outcomes in patients with heart failure with preserved ejection fraction: insights from RELAX. Am $J$ Med 133:e716-e721

European Medicines Agency (2013) Guideline on the investigation of drug interactions.

Fitz-Patrick D, Roberson K, Niwa K, Fujimura T, Mori K, Hall J, Yan X, Shen Z, Liu S, Ito Y, et al. (2019) Safety and efficacy of verinurad, a selective URAT1 inhibitor, for the treatment of patients with gout and/or asymptomatic hyperuricemia in the United States and Japan: Findings from two phase II trials. Mod Rheumatol 29:1042-1052.

Food and Drug Administration (2020) In vitro drug interaction studies - cytochrome P450 enzyme- and transporter-mediated drug interactions: guidance for industry.

Fredlund L, Winiwarter S, and Hilgendorf C (2017) In vitro intrinsic permeability: a transporter-independent measure of caco-2 cell permeability in drug design and development. Mol Pharm 14:1601-1609.

Hall J, Gillen M, Liu S, Miner JN, Valdez S, Shen Z, and Lee C (2018) Pharmacokinetics, pharmacodynamics, and tolerability of verinurad, a selective uric acid reabsorption inhibitor, in healthy Japanese and non-Asian male subjects. Drug Des Devel Ther 12:1799-1807.

Hillgren KM, Keppler D, Zur AA, Giacomini KM, Stieger B, Cass CE, and Zhang L; International Transporter Consortium (2013) Emerging transporters of clinical importance: an update from the International Transporter Consortium. Clin Pharmacol Ther 94:52-63.

Japanese Pharmaceuticals and Medical Devices Agency (2018) Guideline on drug interaction for drug development and appropriate provision of information.

Kalgutkar A and Daniels J (2010) Carboxylic acids and their bioisosteres, in Metabolism, Pharmacokinetics and Toxicity of Functional Groups: Impact of Chemical Building Blocks on ADMET (Smith D, ed) pp 99-154, Royal Society of Chemistry.

Kankam M, Hall J, Gillen M, Yang X, Shen Z, Lee C, Liu S, Miner JN, Walker S, Clauson V, et al. (2018) Pharmacokinetics, pharmacodynamics, and tolerability of concomitant multiple dose administration of verinurad (RDEA3170) and allopurinol in adult male subjects with gout. J Clin Pharmacol 58:1214-1222.

Kim S-J, Yoshikado T, Ieiri I, Maeda K, Kimura M, Irie S, Kusuhara H, and Sugiyama Y (2016) Clarification of the mechanism of clopidogrel-mediated drug-drug interaction in a clinical cassette small-dose study and its prediction based on in vitro information. Drug Metab Dispos 44:1622-1632.

Kimoto E, Chupka J, Xiao Y, Bi YA, and Duignan DB (2011) Characterization of digoxin uptake in sandwich-cultured human hepatocytes. Drug Metab Dispos 39:47-53.

Kimoto E, Vourvahis M, Scialis RJ, Eng H, Rodrigues AD, and Varma MVS (2019) Mechanistic evaluation of the complex drug-drug interactions of maraviroc: contribution of cytochrome P450 3A, P-glycoprotein and organic anion transporting polypeptide 1B1. Drug Metab Dispos 47:493-503.

Lee CA, Yang C, Shah V, Shen Z, Wilson DM, Ostertag TM, Girardet J-L, Hall J, and Gillen M (2018) Metabolism and disposition of verinurad, a uric acid reabsorption inhibitor, in humans. Drug Metab Dispos 46:532-541.

Ma Y, Fu Y, Khojasteh SC, Dalvie D, and Zhang D (2017) Glucuronides as potential anionic substrates of human cytochrome P450 2C8 (CYP2C8). J Med Chem 60:8691-8705. 
Marschall HU, Wagner M, Zollner G, Fickert P, Diczfalusy U, Gumhold J, Silbert D, Fuchsbichler A, Benthin L, Grundström R, et al. (2005) Complementary stimulation of hepatobiliary transport and detoxification systems by rifampicin and ursodeoxycholic acid in humans. Gastroenterology 129:476-485.

Niemi M, Arnold KA, Backman JT, Pasanen MK, Gödtel-Armbrust U, Wojnowski L, Zanger UM, Neuvonen PJ, Eichelbaum M, Kivistö KT, et al. (2006) Association of genetic polymorphism in $\mathrm{ABCC} 2$ with hepatic multidrug resistance-associated protein 2 expression and pravastatin pharmacokinetics. Pharmacogenet Genomics 16:801-808.

Ogilvie BW, Zhang D, Li W, Rodrigues AD, Gipson AE, Holsapple J, Toren P, and Parkinson A (2006) Glucuronidation converts gemfibrozil to a potent, metabolismdependent inhibitor of CYP2C8: implications for drug-drug interactions. Drug Metab Dispos 34:191-197.

Oscarson M, Burk O, Winter S, Schwab M, Wolbold R, Dippon J, Eichelbaum M, and Meyer UA (2007) Effects of rifampicin on global gene expression in human small intestine. Pharmacogenet Genomics 17:907-918.

Ouk S (2013) United States Patent: US 8.541,589 99 B2.

Patel CG, Ogasawara K, and Akhlaghi F (2013) Mycophenolic acid glucuronide is transported by multidrug resistance-associated protein 2 and this transport is not inhibited by cyclosporine, tacrolimus or sirolimus. Xenobiotica 43:229-235.

Picard N (2013) The pharmacokinetic interaction between mycophenolic acid and cyclosporine revisited: a commentary on "Mycophenolic acid glucuronide is transported by multidrug resistance-associated protein 2 and this transport is not inhibited by cyclosporine, tacrolimus or sirolimus". Xenobiotica 43:836-838.

Ramirez-Sandoval JC and Madero M (2018) Treatment of Hyperuricemia in Chronic Kidney Disease, in Uric Acid in Chronic Kidney Disease Contrib Nephrol (TreviñoBecerra AIK, ed) pp 135-146, Swizterland, Basel.

Rodrigues AD, Lai Y, Shen H, Varma MVS, Rowland A, and Oswald S (2020) Induction of human intestinal and hepatic organic anion transporting polypeptides: where is the evidence for its relevance in drug-drug interactions? Drug Metab Dispos 48:205-216.

Sane RS, Ramsden D, Sabo JP, Cooper C, Rowland L, Ting N, Whitcher-Johnstone A, and Tweedie DJ (2016) Contribution of major metabolites toward complex drug drug interactions of deleobuvir: in vitro predictions and in vivo outcomes. Drug Metab Dispos 44:466-475.

Sato Y, Feig DI, Stack AG, Kang DH, Lanaspa MA, Ejaz AA, Sánchez-Lozada LG, Kuwabara M, Borghi C, and Johnson RJ (2019) The case for uric acid-lowering treatment in patients with hyperuricaemia and CKD. Nat Rev Nephrol 15:767-775.

Shen Z, Gillen M, Miner JN, Bucci G, Wilson DM, and Hall JW (2017) Pharmacokinetics, pharmacodynamics, and tolerability of verinurad, a selective uric acid reabsorption inhibitor, in healthy adult male subjects. Drug Des Devel Ther 11:2077-2086.

Shiramoto M, Liu S, Shen Z, Yan X, Yamamoto A, Gillen M, Ito Y, and Hall J (2018) Verinurad combined with febuxostat in Japanese adults with gout or asymptomatic hyperuricaemia: a phase 2a, open-label study. Rheumatology (Oxford) 57:1602-1610.

Shitara Y, Hirano M, Sato H, and Sugiyama Y (2004) Gemfibrozil and its glucuronide inhibit the organic anion transporting polypeptide 2 (OATP2/OATP1B1:SLC21A6)mediated hepatic uptake and CYP2C8-mediated metabolism of cerivastatin: analysis of the mechanism of the clinically relevant drug-drug interaction between cerivastatin and gemfibrozil. J Pharmacol Exp Ther 311:228-236.

Smith WB, Hall J, Berg JK, Kazimir M, Yamamoto A, Walker S, Lee CA, Shen Z, Wilson DM, Zhou D, et al. (2018) Effect of renal impairment on the pharmacokinetics and pharmacodynamics of verinurad, a selective uric acid reabsorption inhibitor. Clin Drug Investig 38:703-713.

Suzuki H and Sugiyama Y (2002) Single nucleotide polymorphisms in multidrug resistance associated protein 2 (MRP2/ABCC2): its impact on drug disposition. Adv Drug Deliv Rev 54:1311-1331.

Tan PK, Liu S, Gunic E, and Miner JN (2017) Discovery and characterization of verinurad, a potent and specific inhibitor of URAT1 for the treatment of hyperuricemia and gout. Sci Rep 7:665.

Terkeltaub R, Dronamraju N, Johansson SA, Parkinson J, Johnsson E, Erlandsson F, and Stack A (2019) OP0207 Urate-lowering therapy with verinurad and febuxostat reduces serum uric acid and albuminuria in hyperuricemic patients with diabetes. Ann Rheum Dis 78:179-180.

Tornio A, Filppula AM, Kailari O, Neuvonen M, Nyrönen TH, Tapaninen T, Neuvonen PJ, Niemi M, and Backman JT (2014) Glucuronidation converts clopidogrel to a strong time-dependent inhibitor of CYP2C8: a phase II metabolite as a perpetrator of drug-drug interactions. Clin Pharmacol Ther 96:498-507.

Varma MV, Pang KS, Isoherranen N, and Zhao P (2015) Dealing with the complex drug-drug interactions: towards mechanistic models. Biopharm Drug Dispos 36:71-92.

Westley IS, Brogan LR, Morris RG, Evans AM, and Sallustio BC (2006) Role of Mrp2 in the hepatic disposition of mycophenolic acid and its glucuronide metabolites: effect of cyclosporine. Drug Metab Dispos 34:261-266.

Yu RZ, Warren MS, Watanabe T, Nichols B, Jahic M, Huang J, Burkey J, Geary RS, Henry SP, and Wang Y (2016) Lack of interactions between an antisense oligonucleotide with 2'-O-(2-Methoxyethyl) modifications and major drug transporters. Nucleic Acid Ther 26:111-117.

Zhang L, Huang S-M, Reynolds K, Madabushi R, and Zineh I (2018) Transporters in drug development: scientific and regulatory considerations. Clin Pharmacol Ther 104:793-796.

Zhang Y, Han Y-H, Putluru SP, Matta MK, Kole P, Mandlekar S, Furlong MT, Liu T, Iyer RA, Marathe P, et al. (2016) Diclofenac and its acyl glucuronide: determination of in vivo exposure in human subjects and characterization as human drug transporter substrates in vitro. Drug Metab Dispos 44:320-328.

Zhang Y, Warren MS, Zhang X, Diamond S, Williams B, Punwani N, Huang J, Huang $\mathrm{Y}$, and Yeleswaram S (2015) Impact on creatinine renal clearance by the interplay of multiple renal transporters: a case study with INCB039110. Drug Metab Dispos 43:485-489.

Address correspondence to: V. Sashi Gopaul, Biopharmaceuticals R\&D, AstraZeneca Gothenburg, Pepparedsleden 1, 43150 Mölndal, Sweden. E-mail: sashi.gopaul@astrazeneca.com 